\title{
Impaired Postnatal Myelination in a Conditional Knockout Mouse for the Ferritin Heavy Chain in Oligodendroglial Cells
}

\author{
Rensheng Wan, ${ }^{\star}$ Veronica T. Cheli,* Diara A. Santiago-González, ${ }^{\star}$ Shaina L. Rosenblum, Qiuchen Wan, and \\ (D) Pablo M. Paez \\ Hunter James Kelly Research Institute, Department of Pharmacology and Toxicology, Jacobs School of Medicine and Biomedical Sciences, State \\ University of New York, University at Buffalo, Buffalo, New York 14203
}

To define the importance of iron storage in oligodendrocyte development and function, the ferritin heavy subunit (Fth) was specifically deleted in oligodendroglial cells. Blocking Fth synthesis in Sox10 or NG2-positive oligodendrocytes during the first or the third postnatal week significantly reduces oligodendrocyte iron storage and maturation. The brain of Fth KO animals presented an important decrease in the expression of myelin proteins and a substantial reduction in the percentage of myelinated axons. This hypomyelination was accompanied by a decline in the number of myelinating oligodendrocytes and with a reduction in proliferating oligodendrocyte progenitor cells (OPCs). Importantly, deleting Fth in Sox10-positive oligodendroglial cells after postnatal day 60 has no effect on myelin production and/or oligodendrocyte quantities. We also tested the capacity of Fth-deficient OPCs to remyelinate the adult brain in the cuprizone model of myelin injury and repair. Fth deletion in NG2-positive OPCs significantly reduces the number of mature oligodendrocytes and myelin production throughout the remyelination process. Furthermore, the corpus callosum of Fth $\mathrm{KO}$ animals presented a significant decrease in the percentage of remyelinated axons and a substantial reduction in the average myelin thickness. These results indicate that Fth synthesis during the first three postnatal weeks is important for an appropriate oligodendrocyte development, and suggest that Fth iron storage in adult OPCs is also essential for an effective remyelination of the mouse brain.

Key words: ferritin; iron; myelination; oligodendrocytes; remyelination

Significance Statement

To define the importance of iron storage in oligodendrocyte function, we have deleted the ferritin heavy chain (Fth) specifically in the oligodendrocyte lineage. Fth ablation in oligodendroglial cells throughout early postnatal development significantly reduces oligodendrocyte maturation and myelination. In contrast, deletion of $F$ th in oligodendroglial cells after postnatal day 60 has no effect on myelin production and/or oligodendrocyte numbers. We have also tested the consequences of disrupting Fth iron storage in oligodendrocyte progenitor cells (OPCs) after demyelination. We have found that Fth deletion in NG2-positive OPCs significantly delays the remyelination process in the adult brain. Therefore, Fth iron storage is essential for early oligodendrocyte development as well as for OPC maturation in the demyelinated adult brain.

Received May 22, 2020; revised Aug. 14, 2020; accepted Aug. 19, 2020.

Author contributions: R.W., V.T.C., D.A.S.-G., and P.M.P. designed research; R.W., V.T.C., D.A.S.-G., S.L.R., Q.W., and P.M.P. performed research; R.W., V.T.C., D.A.S.-G., S.L.R., Q.W., and P.M.P. analyzed data; R.W.,

V.T.C., D.A.S.-G., and P.M.P. wrote the first draft of the paper; V.T.C., D.A.S.-G., and P.M.P. edited the paper;

V.T.C., D.A.S.-G., and P.M.P. wrote the paper.

*R.W., V.T.C., and D.A.S.-G. contributed equally to this work.

This work was supported by National Institute of Neurological Disorders and Stroke Award R01NS07804 and Jacobs School of Medicine and Biomedical Sciences, University at Buffalo (startup package).

The authors declare no competing financial interests.

Correspondence should be addressed to Pablo M. Paez at ppaez@buffalo.edu.

https://doi.org/10.1523/JNEUROSCI.1281-20.2020

Copyright $\odot 2020$ the authors

\section{Introduction}

Oligodendrocyte development, myelin production, and iron homeostasis are closely connected. In the CNS, iron is essential for myelin synthesis and maintenance (Todorich et al., 2009; Cheli et al., 2018). Iron is directly required for myelin production as a cofactor for enzymes involved in cholesterol and lipid synthesis (Beard et al., 2003), and oligodendrocytes are the cells with the highest iron levels in the brain, which is linked to their highly metabolic needs associated with the process of myelination (LeVine and Macklin, 1990; Connor and Menzies, 1996). Iron deficiency leads to hypomyelination, both in animal models and humans, and the neurologic consequences of iron deficiency during early brain development are permanent. For example, 
rodents subjected to iron deficiency during development exhibit impaired oligodendrocyte progenitor cell (OPC) proliferation and differentiation, as well as reduced synthesis of myelin proteins (Rosato-Siri et al., 2010). Iron deficiency also leads to altered myelin composition characterized by reduced levels of cholesterol, galactolipids, phospholipids, and myelin proteins (Badaracco et al., 2008).

Cell iron equilibrium is precisely orchestrated by several proteins, which work coordinately to regulate iron uptake and storage. Ferritin is the main intracellular iron-storage protein and is crucial for keeping iron in a nontoxic form. Ferritin is a 24-subunit heteropolymer, composed of heavy (Fth) and light (Ftl) chains, capable of binding $>4500$ atoms of iron (Arosio et al., 2009), which can be mobilized for metabolic purposes through lysosomal turnover (Y. Zhang et al., 2010). The Fth subunit contains ferroxidase activity, which is required for converting ferrous iron into ferric iron, which is then deposited inside the ferritin core (Arosio et al., 2009; Bou-Abdallah, 2010). The expression of ferritin in the rodent brain is highest at birth and declines after the peak of myelination (Todorich et al., 2009). Both Fth and Ftl were found to be significantly upregulated during oligodendrocyte maturation in vitro as well as in vivo (Li et al., 2013; Y. Zhang et al., 2014), and it has been shown that Fth expression precedes iron accumulation in premyelinating oligodendrocytes of the developing brain (Ortiz et al., 2004; Schonberg et al., 2012; Li et al., 2013). However, the role of Fth in oligodendrocyte development and myelination has not been defined.

In this work, we have found that Fth ablation in oligodendroglial cells significantly affects the myelination of the postnatal brain. Fth $\mathrm{KO}$ mice display an important decrease in the numbers of mature oligodendrocytes and myelin synthesis. This suggests that oligodendrocytes need to express Fth through early postnatal development to store adequate quantities of iron to complete the maturational process. In contrast, our results indicate that oligodendroglial cells do not require to express Fth to maintain and/or renovate the myelin sheath after postnatal day 60. Additionally, Fth deletion in OPCs significantly delays the remyelination process, suggesting that OPCs of the adult brain also need to incorporate and store iron in Fth to properly remyelinate the CNS.

\section{Materials and Methods}

Transgenic mice. All animals used in the present study were housed in the University at Buffalo Division of Laboratory Animal Medicine vivarium, and procedures were approved by University at Buffalo's Animal Care and Use Committee, and conducted in accordance with the guidelines in Guide for the care and use of laboratory animals from the National Institutes of Health. The heterozygous floxed Fth mice (Darshan et al., 2009) (JAX stock \#018063), the Sox10CreER ${ }^{\mathrm{T} 2}$ mice (Matsuoka et al., 2005) (JAX stock \#025807), and the NG2Cre $\mathrm{ER}^{\mathrm{T} 2}$ transgenic line (Zhu et al., 2011) (JAX stock \#008538) were obtained from The Jackson Laboratory. Experimental animals were generated in our laboratory by crossing the heterozygous floxed Fth line with the hemizygous Sox10CreER ${ }^{\mathrm{T} 2}$ or NG2CreER $\mathrm{ER}^{\mathrm{T2}}$ transgenic lines. For all the experiments presented in this work, mice of either sex were used.

Mice treatments. To delete Fth in Sox10- or NG2-positive oligodendrocytes, Cre activity was induced starting at postnatal day 2 (P2). P2 Sox10$\mathrm{Fth}^{\mathrm{KO}}\left(\mathrm{Fth}^{\mathrm{f} / \mathrm{f}}\right.$, Sox10CreER $\left.{ }^{\mathrm{Cre} /}-\right), \mathrm{NG} 2-\mathrm{Fth}^{\mathrm{KO}}\left(\mathrm{Fth}^{\mathrm{f} / \mathrm{f}}, \mathrm{NG}^{\mathrm{CCreER}}{ }^{\mathrm{Cre} /-}\right)$ and control (Cre-negative) littermates (Fth ${ }^{\mathrm{f} / \mathrm{f}}$, Sox10Cre $\mathrm{ER}^{-/-}$or NG2CreER ${ }^{-/-}$) were intraperitoneally injected once a day for 5 consecutive days with $25 \mathrm{mg} /$ $\mathrm{kg}$ tamoxifen (Sigma Millipore), and brain tissue was collected at P15, P30, and P60. Furthermore, P60 Sox10-Fth ${ }^{\mathrm{KO}}$ and control littermates were injected intraperitoneally once a day for 5 consecutive days with $100 \mathrm{mg} / \mathrm{kg}$ tamoxifen, and brain tissue was collected at P90. For remyelination studies, P60 NG2-Fth ${ }^{\mathrm{KO}}\left(\mathrm{Fth}^{\mathrm{f} / \mathrm{f}}, \mathrm{NG} 2 \mathrm{CreER}{ }^{\mathrm{Cre} /-}\right.$ ) and control (Cre negative) littermates $\left(\mathrm{Fth}^{\mathrm{f} / \mathrm{f}}, \mathrm{NG} 2 \mathrm{CreER}{ }^{-I-}\right.$ ) were fed pellet chow containing $0.2 \%$ cuprizone (CPZ; Teklad-Envigo) for 7 weeks and were injected with $100 \mathrm{mg} / \mathrm{kg}$ tamoxifen every other day during the last 2 weeks of the CPZ treatment (Santiago-González et al., 2017). Additionally, a group of control (Fth ${ }^{\mathrm{f} / \mathrm{f}}$, $\mathrm{NG} 2 \mathrm{CreER}{ }^{-I}$ ) mice were maintained on a diet of normal pellet chow.

Primary cultures of cortical OPCs. Primary cultures of cortical OPCs were prepared as described by Cheli et al. (2015). First, cerebral hemispheres from 1-d-old mice were mechanically dissociated and were plated on poly-D-lysine-coated flasks in DMEM/F12 (1:1 v/v) (Invitrogen), supplemented with 10\% FBS (Invitrogen). After $4 \mathrm{~h}$, the medium was changed and the cells were grown in DMEM/F12 supplemented with insulin $(5 \mu \mathrm{g} / \mathrm{ml})$, apo-transferrin $(50 \mu \mathrm{g} / \mathrm{ml})$, sodium selenite (30 nM), D-biotin (10 mM), and 10\% FBS (Invitrogen). Two-thirds of the culture media were changed every $3 \mathrm{~d}$. After $14 \mathrm{~d}$, OPCs were purified from the mixed glial culture by a differential shaking and adhesion procedure and allowed to grow on poly-D-lysine-coated coverslips in DMEM/F12 supplemented with insulin $(5 \mu \mathrm{g} / \mathrm{ml})$, apo-transferrin $(50 \mu \mathrm{g} / \mathrm{ml})$, sodium selenite (30 nM), $0.1 \%$ BSA, progesterone $(0.06 \mathrm{ng} /$ $\mathrm{ml})$, and putrescine $(16 \mu \mathrm{g} / \mathrm{ml})$ (Sigma Millipore). OPCs were kept in mitogens, PDGF and bFGF (20 ng/ml) (PeproTech), for $2 \mathrm{~d}$ and then induced to differentiate by mitogen withdrawal and T3 (15 nM) addition. Because of the inherent variability in cell growth from culture to culture, quadruplicate cultures were prepared. Since the size of the litter significantly affects pup's development, litters with $<5$ or $>10$ pups were not used.

Immunocytochemistry. Cells were stained with antibodies against several oligodendrocyte stage-specific markers following the protocol outlined by Cheli et al. (2016). Fluorescent images were obtained using a spinning disk confocal microscope (Olympus, IX83-DSU). Quantitative analysis of the results was done counting the antigen-positive and DAPIpositive cells (total number of cells) in 20 randomly selected fields per coverslip, which resulted in counts of $>2000$ cells. For all experimental conditions, four coverslips per culture were analyzed, and data represent pooled results from at least four independent cultures. Cell counting was performed semiautomatically and blind to the genotype of the sample by MetaMorph software (Molecular Devices). The primary antibodies used for immunocytochemistry were as follows: CC1 (mouse; 1:300; Calbiochem), MBP (mouse; 1:400; Covance), NG2 (rabbit; 1:400; Millipore), Olig1 (mouse; 1:400; Chemi-Con), Olig2 (mouse and rabbit; 1:500; Millipore), and PLP (rat; 1:1000; AA3-PLP/DM20).

Western blot. Protein samples were extracted using lysis buffer as described by Santiago-González et al. (2017); $20 \mu \mathrm{g}$ of proteins was separated with NuPAGE Novex 4\%-12\% Bis-Tris Protein Gels (Invitrogen) and electroblotted onto PVDF membranes. Membranes were blocked overnight at $4^{\circ} \mathrm{C}$ with $5 \%$ nonfat milk, $0.1 \%$ Tween-20 in PBS. Primary antibodies were diluted in a blocking solution and membranes incubated $3 \mathrm{~h}$ at room temperature with agitation. Protein bands were detected by chemiluminescence using the ECL kit (GE Healthcare) with HRP-conjugated secondary antibodies (GE Healthcare) and scanned with a C-Digit Bot Scanner (LI-COR). Protein bands were quantified using the Image Studio Software (LI-COR). The primary antibodies used were as follows: CNP (1:1000; Neo-Markes), MBP (mouse; 1:1000; Covance), MOG (mouse; 1:1000; Millipore), PLP (rat; 1:500, AA3-PLP/DM20), and P84 (mouse; 1:10,000; GeneTex).

$R T-P C R$. Total RNA was isolated using Trizol reagent (Invitrogen). RNA content was estimated by measuring the absorbance at $260 \mathrm{~nm}$, and the purity was assessed by assessing the ratio of absorbance: $260 /$ $280 \mathrm{~nm}$. PCR primers were designed based on published sequences (Darshan et al., 2009). First-strand cDNA was prepared from $1 \mu \mathrm{g}$ of total RNA using SuperScript III RNase H-reverse transcriptase (Invitrogen) and $1 \mu \mathrm{g}$ of oligo(dT). The mRNA samples were denaturized at $65^{\circ} \mathrm{C}$ for $5 \mathrm{~min}$. Reverse transcription was performed at $50^{\circ} \mathrm{C}$ for $55 \mathrm{~min}$ and was stopped by heating the samples at $85^{\circ} \mathrm{C}$ for $5 \mathrm{~min}$. The cDNA was amplified by PCR using specific primers and PCR Platinum Supermix reagent (Invitrogen). PCR conditions were as follows: $94^{\circ} \mathrm{C}$ for $2 \mathrm{~min}, 40$ cycles of $94^{\circ} \mathrm{C}$ for $30 \mathrm{~s}, 58^{\circ} \mathrm{C}$ for $30 \mathrm{~s}$ followed by $72^{\circ} \mathrm{C}$ for $2 \mathrm{~min}$. After 40 cycles, samples were incubated at $72^{\circ} \mathrm{C}$ for $5 \mathrm{~min}$. The 
A

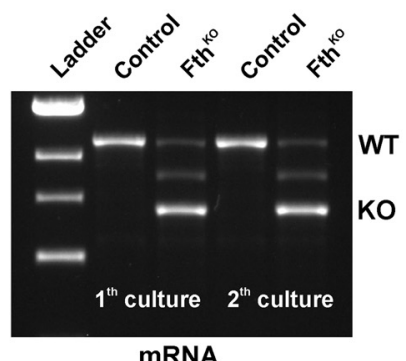

B

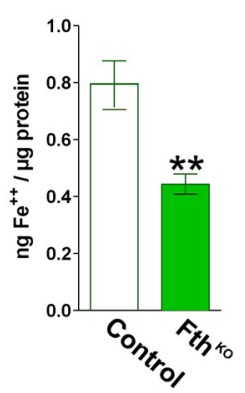

C

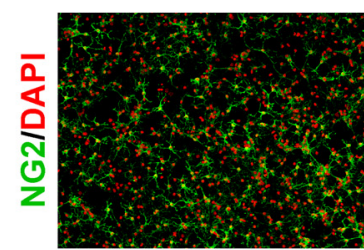

흠

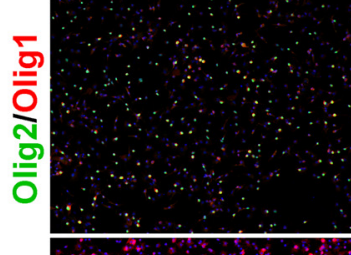

选
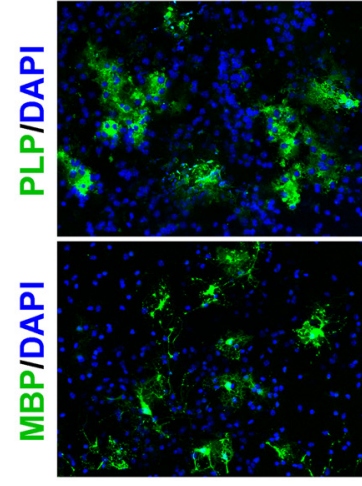

Sox10-Fth ${ }^{\mathrm{kO}}$
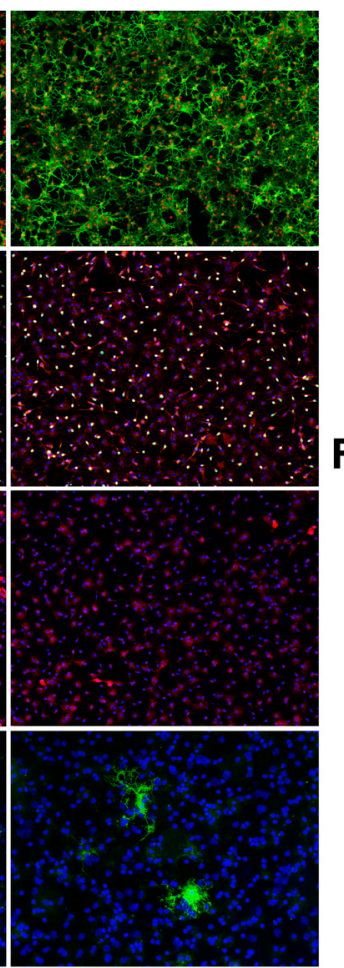

$\mathbf{F}$

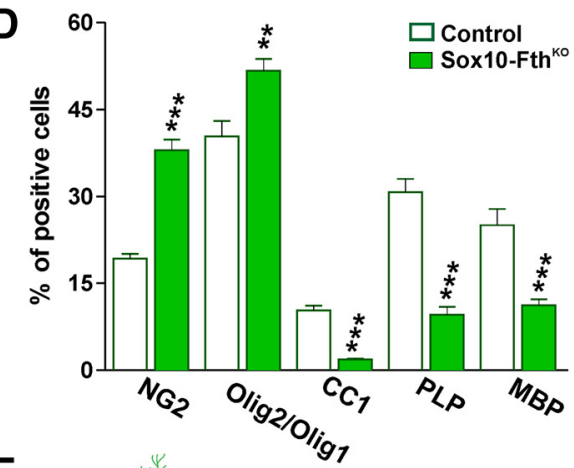

E
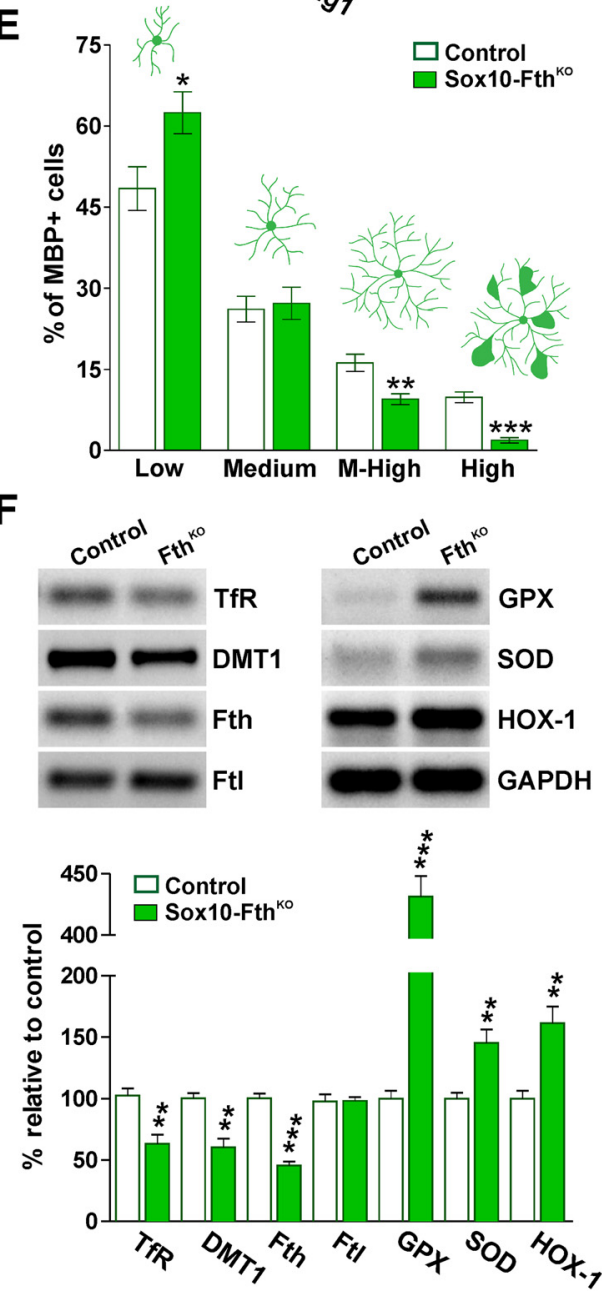

Figure 1. Fth deletion blocks OPC maturation in vitro. $A$, Semiquantitative RT-PCR for the Fth mRNA was performed after 3 days of 4-0H-tamoxifen treatment in control and Sox10-FthKO OPCs. B, Total iron content was examined in control and Sox10-FthK0 OPCs using a colorimetric iron assay. C, D, Two days after mitogen withdrawal, control and Sox 10-FthK0 OPCs were stained with antibodies against NG2, Olig2/0lig1, CC1, PLP, and MBP, and the percentage of positive cells in each experimental condition was examined by confocal microscopy. Scale bar, 80 $\mu \mathrm{m}$. $\boldsymbol{E}$, Morphologic complexity of MBP-positive cells was scored in four categories. $\boldsymbol{F}$, Semiquantitative RT-PCRs for the transferrin receptor (TfR), the divalent metal transporter 1 (DMT1), the ferritin heavy and light chains (Fth, Ftl), the glutathione peroxidase (GPX), the superoxide dismutase (SOD), and the heme oxygenase-1 (H0-1) were performed in control and Sox10-FthKO OPCs $2 \mathrm{~d}$ after mitogen withdrawal. GAPDH was used as the internal standard. Data are summarized based on the relative spot intensities and plotted as percent of controls. Comparisons between experimental groups were made by the unpaired $t$ test. Values are mean \pm SEM of four independent experiments. ${ }^{*} p<0.05 ;{ }^{* *} p<0.01$; ${ }^{* * *} p<0.001$ versus respective controls.

PCR products were visualized on a SYBR Safe-stained agarose gel and the bands digitized using a Gel Doc EZ System (Bio-Rad).

Colorimetric iron assay. The Quantichrom iron assay kit (BioAssay Systems) was used to measure total cellular iron. Briefly, $50 \mu \mathrm{l}$ of samples containing $1 \times 10^{6}$ cells was mixed with $200 \mu$ l Quantichrom working reagent in a 96-well plate and incubated at room temperature for $40 \mathrm{~min}$. The optical density (OD) at $590 \mathrm{~nm}$ was measured by a microplate reader. The OD against standard iron concentrations was plotted by subtracting blank (water) OD from the standard OD values, and the slope of the data plot then determined using linear regression. The total protein concentration was estimated using the Pierce BCA Protein Assay Kit (Thermo Fisher Scientific).

Immunohistochemistry. All animals were anesthetized with isoflurane and then perfused with $4 \%$ of PFA in PBS via the left ventricle. The brains were postfixed overnight in the same fixative solution at $4^{\circ} \mathrm{C}$. Coronal brain slices of $50 \mu \mathrm{m}$ thick were obtained using a vibratome (Leica Biosystems, VT1000-S). Free-floating vibratome sections were incubated in a blocking solution ( $2 \%$ normal goat serum and $1 \%$ Triton $\mathrm{X}-100$ in PBS) for $2 \mathrm{~h}$ at room temperature and then incubated with the primary antibody overnight at $4^{\circ} \mathrm{C}$. Sections were then rinsed in PBS 
A Control
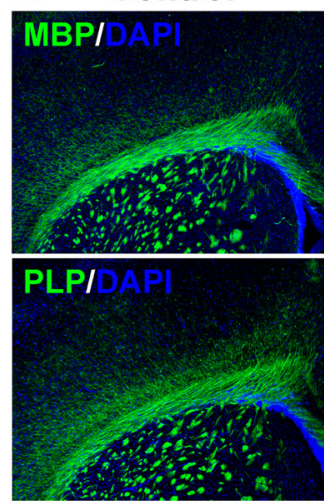

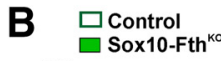

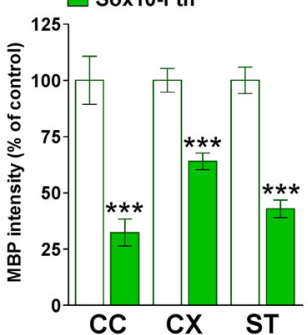

c

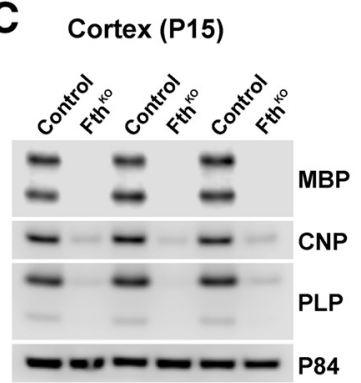

D Cerebellum (P15)

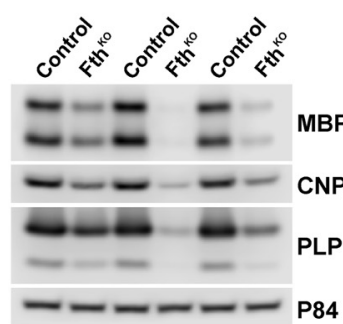

Sox10-Fth ${ }^{\mathrm{KO}}$
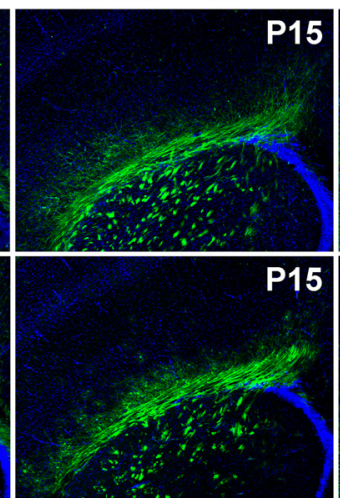

P15
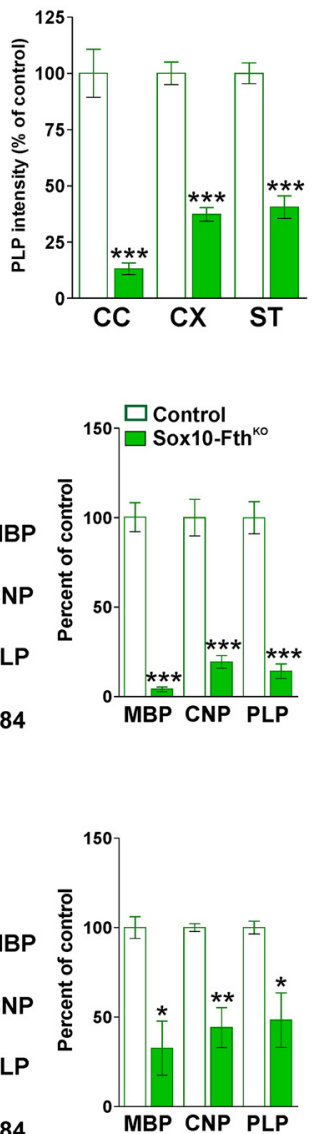

Control
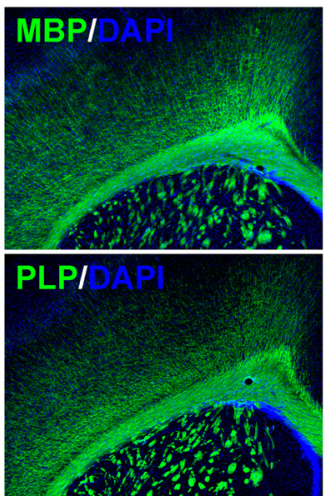

P30

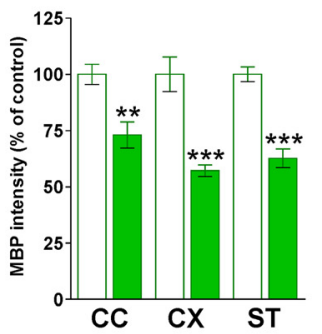

Cortex (P30)
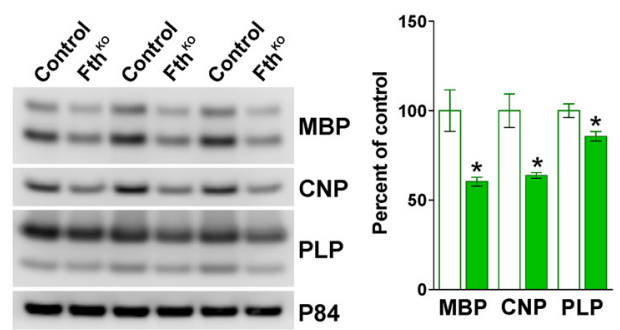

Cerebellum (P30)

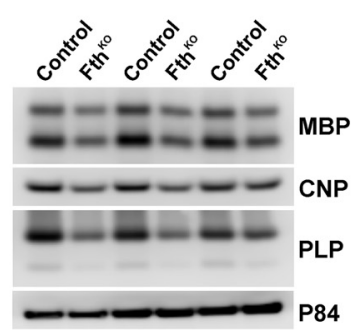

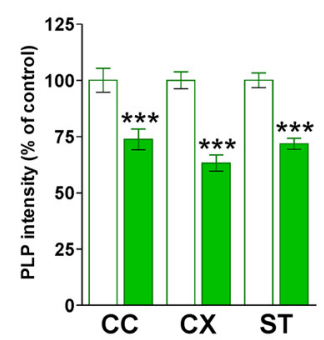

Sox10-Fth ${ }^{\text {KO }}$

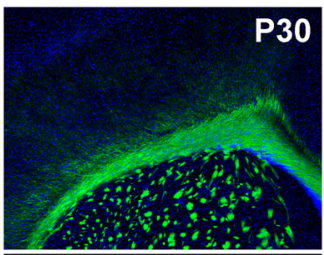

P30

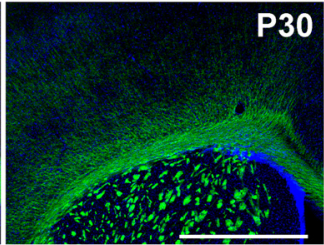

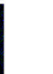


A

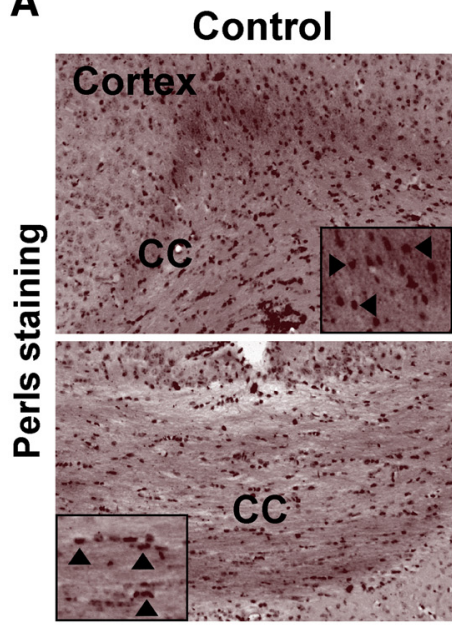

C

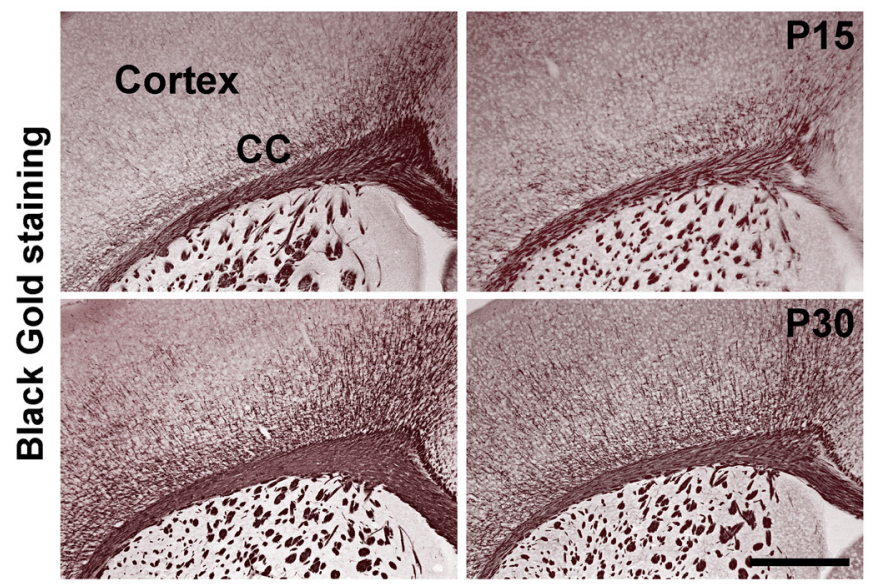

Sox10-Fth ${ }^{\mathrm{KO}}$

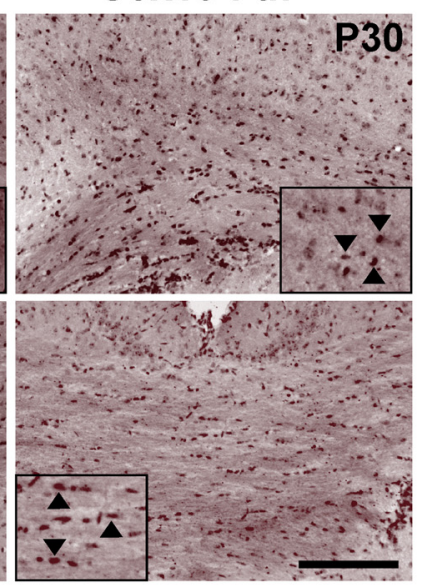

15

B $\square$ Control $\square$ Sox10-Fth ${ }^{\mathrm{ko}}$
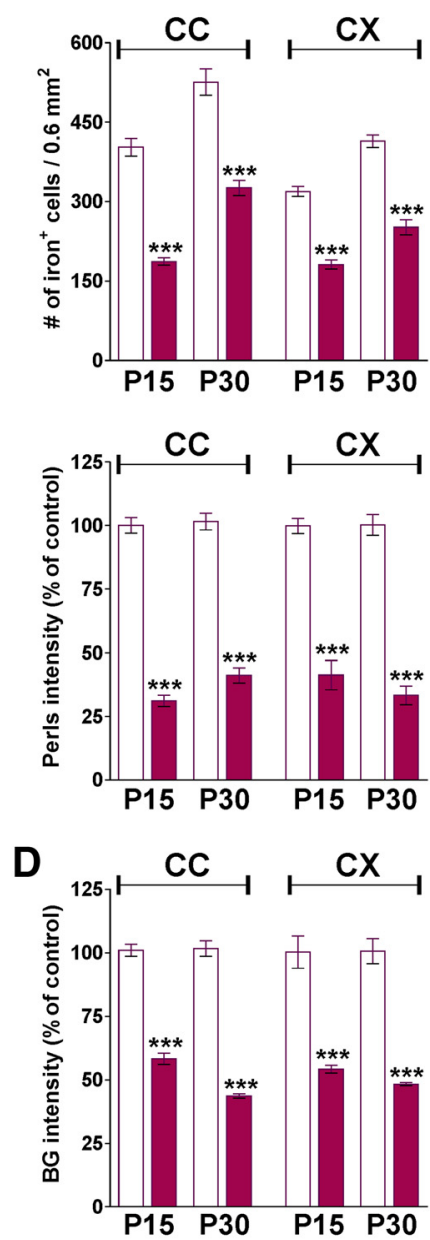

Figure 3. Perls and Black Gold staining in the Sox10-FthK0 brain. A, Perls staining in representative brain coronal sections collected from control and Sox10-FthK0 mice at P30. Arrowheads in high-magnification insets indicate Perls-positive cells that were selected for the analysis. Scale bar, $90 \mu \mathrm{m}$. $\boldsymbol{B}$, The total number of Perls-positive oligodendrocytes and the average intensity staining per cell were quantified in the central area of the corpus callosum $(C C)$ and in the cortex (CX) at P15 and P30. C, Black Gold II staining in representative brain coronal sections collected from control and Sox10-FthK0 mice at P15 and P30. Scale bar, $180 \mu \mathrm{m}$. D, Black Gold II intensity staining was quantified in the central area of the corpus callosum (CC) and in the cortex (CX) at P15 and P30. Comparisons between experimental groups were made by the unpaired $t$ test. Six brains per experimental condition were analyzed. Values are mean \pm SEM. ${ }^{* *} p<0.001$ versus respective controls.

Technology), CC1 (mouse; 1:300; Calbiochem), Ki67 (rabbit; 1:250; Abcam), Ki67 (mouse; 1:250; BD Biosciences), MBP (mouse; 1:1000; Covance), Olig2 (mouse and rabbit; 1:500; Millipore), PLP (rat; 1:250, AA3 - PLP/DM20), and Sox2 (rabbit; 1:200; Millipore).

Black-gold II myelin staining. Black Gold II staining was performed as described by Cheli et al. (2016). Briefly, PFA-fixed brain sections of $50 \mu \mathrm{m}$ were mounted onto Superfrost Plus slide (Thermo Fisher Scientific). Coronal brain slices were initially air dried and then rehydrated and transferred to a lukewarm $0.3 \%$ Black Gold II solution (Millipore). After color development $(\sim 10 \mathrm{~min})$, the slides were rinsed with a $1 \%$ sodium thiosulfate solution at $60^{\circ} \mathrm{C}$, dehydrated, and mounted with Permount. The integrated staining intensity in the corpus callosum and cortex was assessed by MetaMorph software (Molecular Devices). The staining intensity in control mice was given the arbitrary value of 100 , and the staining intensity in Sox 10-Fth ${ }^{\mathrm{KO}}$ mice was determined as a percentage of that in control mice. Seven slices per brain $(50 \mu \mathrm{m}$ each) were used, and data represent pooled results from at least six brains per experimental group.

Perls histochemistry. Enhanced Perls histochemistry was performed as described previously by Cheli et al. (2018). Briefly, $20 \mu \mathrm{m}$ coronal brain sections were incubated with $1 \% \mathrm{H}_{2} \mathrm{O}_{2}$ in methanol for $15 \mathrm{~min}$ and then with $2 \%$ potassium ferrocyanide, $\mathrm{pH} 1.0$, overnight (Iron Stain Kit, Sigma Millipore). The reaction was enhanced for $30 \mathrm{~min}$ with $0.025 \%$ $3,3^{\prime}$-diaminobenzidine- $4 \mathrm{Hl}, 0.05 \% \mathrm{H}_{2} \mathrm{O}_{2}$ and $0.005 \% \mathrm{CoCl}_{2}$ in $0.1 \mathrm{M} \mathrm{PB}$.
Finally, sections were dehydrated and mounted with Permount. The number of positive oligodendrocytes and the integrated staining intensity per cell were assessed by MetaMorph software (Molecular Devices) in the central area of the corpus callosum, between the midline and below the apex of the cingulum $\left(0.6 \mathrm{~mm}^{2}\right)$ and in the motor and cingulate cortex, including M1, M2, Cg1, and Cg2 $\left(0.6 \mathrm{~mm}^{2}\right)$ (Franklin and Paxinos, 2008, their Fig. 24). Moreover, the integrated staining intensity in neurons was assessed in the motor cortex (M1), including all cortical layers (Franklin and Paxinos, 2008, their Fig. 24), in the globus pallidus (Franklin and Paxinos, 2008, their Fig. 42), and in the substantia nigra (Franklin and Paxinos, 2008, their Fig. 52). Twelve slices per brain (20 $\mu \mathrm{m}$ each) were used, and data represent pooled results from at least six brains per experimental group.

Electron microscopy. Mouse brains were perfused transcardially with $3 \%$ PFA and $1 \%$ glutaraldehyde. The body of the corpus callosum at the anterior-dorsal level of the hippocampus was dissected and resin-embedded. Thin sections were stained with uranyl acetate and lead citrate and photographed with a FEI Tecnai F20 transmission electron microscope as previously described (Cheli et al., 2016). For $g$-ratio measurements, at least 150 fibers per animal were analyzed. The percentage of myelinated axons was determined in 10 randomly selected fields per sample, which resulted in counts of $>1000$ axons. The $g$-ratio and the percentage of myelinated axons were determined semiautomatically and blind to the genotype of the sample using MetaMorph software (Molecular Devices). 

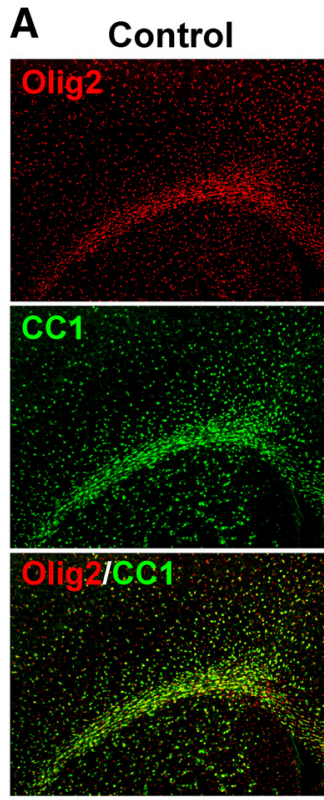

B
Sox10-Fth ${ }^{\mathrm{kO}}$

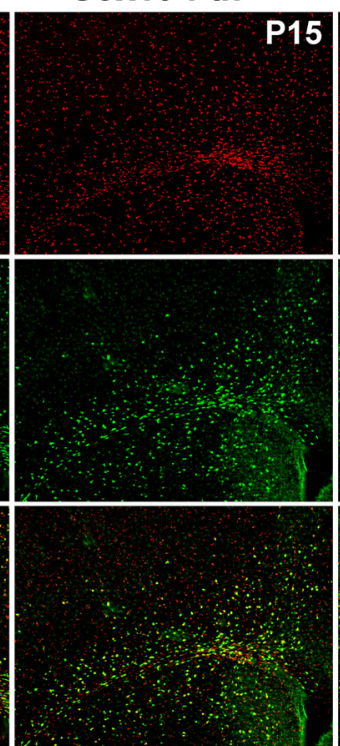

Control
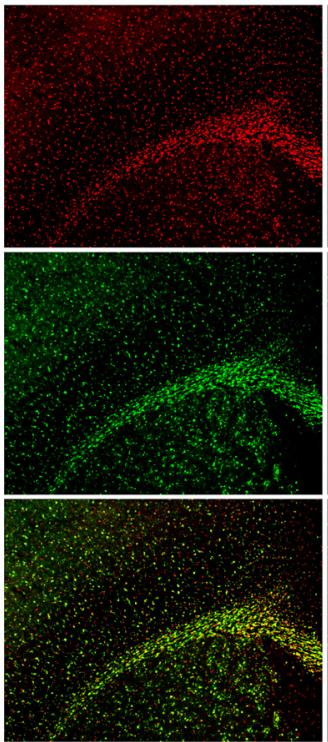

$\square$ Control $\square$ Sox10-Fth ${ }^{\text {Ko }}$

Sox10-Fth ${ }^{\mathrm{kO}}$

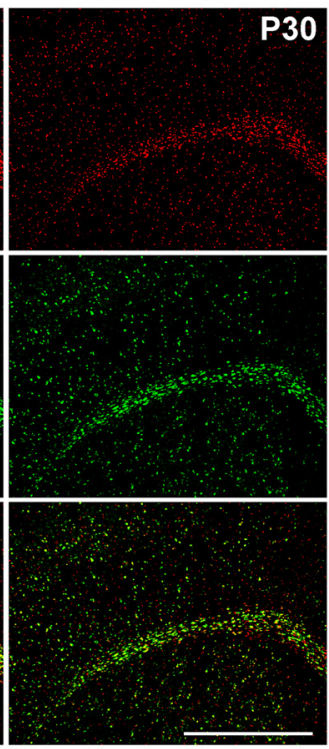

$\square$ Control $\square$ Sox10-Fth ${ }^{\text {Ko }}$
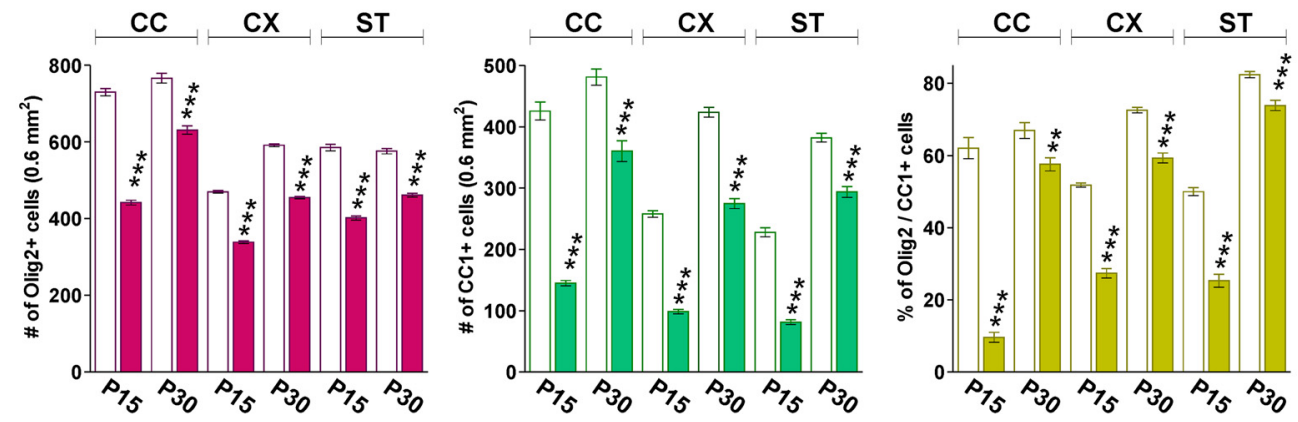

Figure 4. Decreased number of mature oligodendrocytes in the Sox10-FthK0 mouse. $A$, Representative coronal sections from control and Sox10-FthK0 brains at P15 and P30 immunostained for Olig2 and CC1. Scale bar, $180 \mu \mathrm{m}$. B. The total number of Olig2- and C(1-positive cells, and the percentage of 0lig2/CC1 double-positive cells were quantified in the central area of the corpus callosum (CC), in the cortex (CX), and in the striatum (ST) at P15 and P30. Comparisons between experimental groups were made by the unpaired $t$ test. Six brains per experimental condition were analyzed. Values are mean \pm SEM. ${ }^{* *} p<0.01 ;{ }^{* * *} p<0.001$ versus respective controls.

For all experimental conditions, data represent pooled results from at least 4 mice.

Statistical analysis. All datasets were tested for normal distribution using the Kolmogorov-Smirnov test. Single between-group comparisons were made by the unpaired $t$ test (Student's $t$ test), using 95\% CI. Multiple comparisons were investigated by one-way ANOVA followed by Bonferroni's multiple comparison test to detect pairwise betweengroup differences. For the analysis of $g$-ratio scatter plots, simple linear regression with a $95 \% \mathrm{CI}$ was used. All statistical tests were performed in GraphPad Prism (GraphPad Software). A fixed value of $p<0.05$ for two-tailed test was the criterion for reliable differences between groups. Data are presented as mean \pm SEM. To minimize bias, the quantification of all the experiments described in this work was performed blinded to the sample genotype. Based on previous studies and the fact that all comparisons were made between mice with the same genetic background, at least 6 animals for each genotype were compared for all the morphologic and biochemical endpoints.

\section{Results}

\section{Conditional deletion of Fth in cortical OPCs}

A conditional KO mouse for the ferritin heavy chain (Fth) in the oligodendrocyte lineage was created by cross-breeding the floxed mutant Fth mouse (Darshan et al., 2009) with the Sox10CreER ${ }^{\mathrm{T} 2}$ transgenic line (Zhu et al., 2011). Sox10CreER ${ }^{\mathrm{T} 2}$ transgenic mice express a tamoxifen-inducible Cre recombinase under the control of the mouse Sox10 promoter, which postnatally restricts Cre expression to oligodendroglial cells (Zhu et al., 2011; Cheli et al., 2016). Initially, OPCs were isolated from the brain cortices of P1 Fth conditional KO mice (Sox10-Fth ${ }^{\mathrm{KO}}$; $\mathrm{Fth}^{\mathrm{f} / \mathrm{f}}$, Sox10$\mathrm{CreER}{ }^{\mathrm{Cre} /-}$ ) and control (Cre negative) littermates (Control; $\mathrm{Fth}^{\mathrm{f} / \mathrm{f}}$, Sox10-Cre $\mathrm{ER}^{-/-}$) and were kept in a proliferative state in the presence of PDGF and bFGF. After 2 DIV, OPCs were differentiated by mitogens withdrawal and T3 addition. The induction of Cremediated recombination was done by adding 4-OH-tamoxifen to the culture medium during the first 3 DIV. RT-PCR experiments demonstrated high recombination efficiency and reduced Fth mRNAs levels in Fth ${ }^{\mathrm{KO}}$ oligodendrocytes (Fig. 1A). Fth ${ }^{\mathrm{KO}}$ oligodendrocytes displayed a $\sim 50 \%$ reduction in total intracellular iron (Fig. $1 B$ ), and in the expression of myelin proteins, such as MBP and PLP (Fig. 1C,D). Furthermore, the proportion of immature NG2- and Olig1-positive oligodendrocytes was significantly higher in Fth-deficient cultures (Fig. $1 C, D$ ). Suggesting a delay in oligodendrocyte morphologic maturation, a decrease in the percentage of cells with medium-high and high morphology, and an increase in the percentage of cells with low complexity were found in Fthdeficient cultures (Fig. 1E). Thus, oligodendrocytes lacking Fth showed a simple morphology, lower levels of intracellular iron, and less myelin protein expression than controls. Furthermore, we have examined the expression of several proteins implicated in 
A
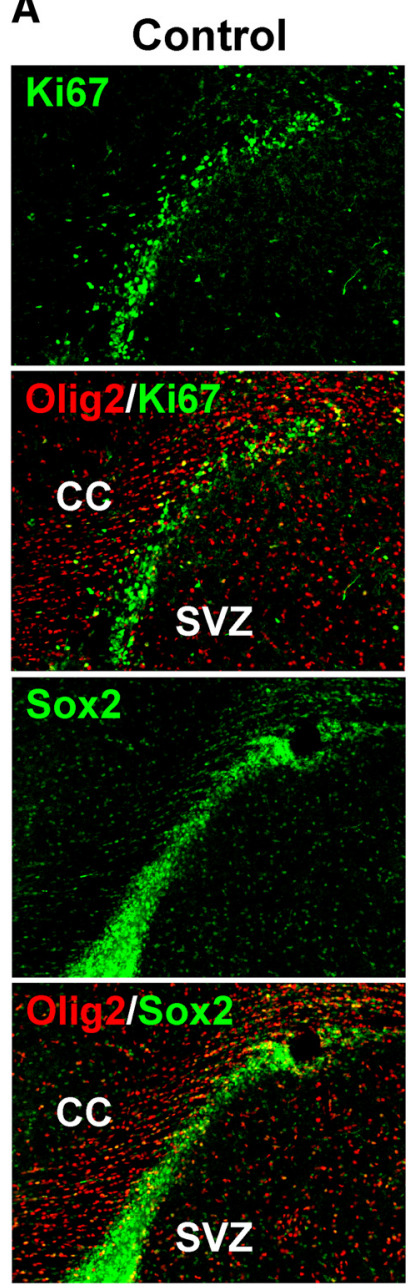
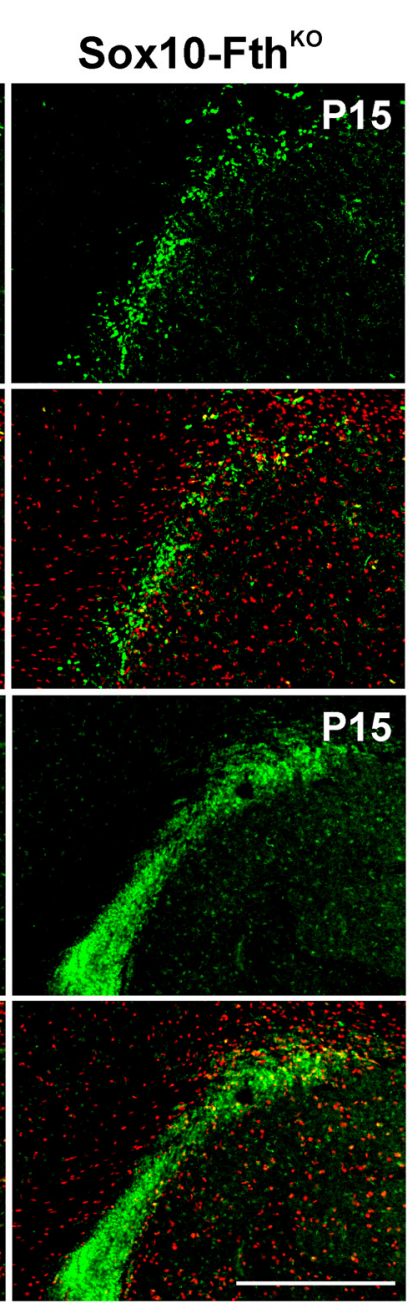

B

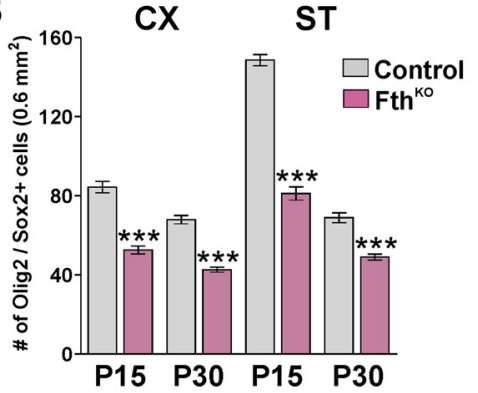

C

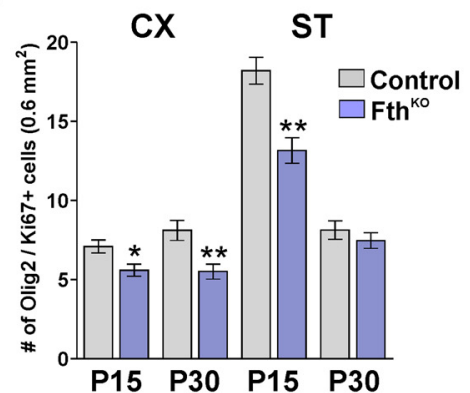

CX

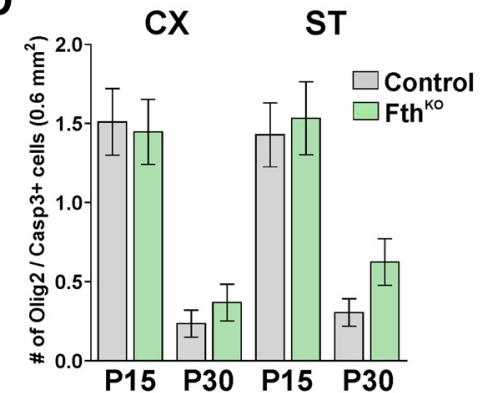

CC

SVZ

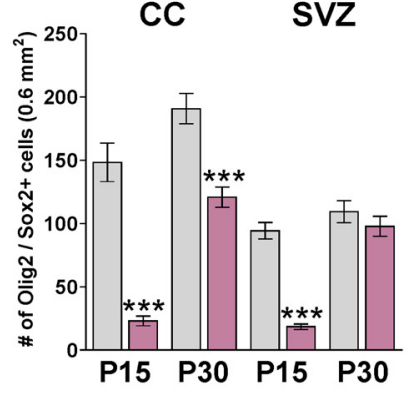

CC

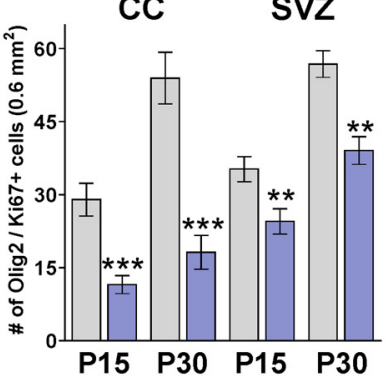

CC

SVZ

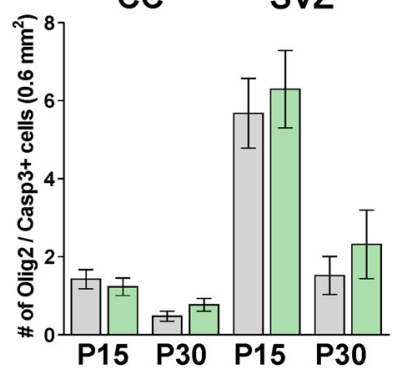

Figure 5. Decreased number of proliferating OPCS in the Sox10-FthK0 brain. $\boldsymbol{A}$, Representative coronal sections from control and Sox10-FthK0 brains at P15 immunostained for 0lig2, Ki67, and Sox2. Scale bar, $90 \mu \mathrm{m}$. B-D, The total number of 0lig2/Sox2, 0lig2/Ki67, and Olig2/Caspase-3 double-positive cells was quantified in the cortex (CX), in the striatum (ST), in the central area of the corpus callosum (CC), and in the subventricular zone (SVZ) at P15 and P30. Comparisons between experimental groups were made by the unpaired $t$ test. Six brains per experimental condition were analyzed. Values are mean \pm SEM. ${ }^{*} p<0.05 ;{ }^{* *} p<0.01 ;{ }^{* * *} p<0.001$ versus respective controls.

oligodendrocyte iron absorption and the synthesis of key antioxidant enzymes by semiquantitative RT-PCR (Fig. $1 F$ ). Proteins implicated in iron uptake, such as the transferrin receptor and the divalent metal transporter 1, were found to be downregulated in $\mathrm{Fth}^{\mathrm{KO}}$ oligodendrocytes (Fig. $1 F$ ). As was expected, the mRNA for Fth was also significantly reduced in $\mathrm{Fth}^{\mathrm{KO}}$ cells; however, the expression of Ftl was equal to control levels (Fig. $1 F$ ). Importantly, the synthesis of the glutathione peroxidase, the superoxide dismutase, and the stressinduced heme oxygenase- 1 was increased in Fth-deficient oligodendrocytes (Fig. 1F). These results indicate the presence of an ironinduced oxidative stress in $\mathrm{Fth}^{\mathrm{KO}}$ oligodendrocytes.

\section{The function of Fth in the postnatal myelination of the} mouse brain

To delete Fth in Sox10-positive oligodendroglial cells, Cre activity was induced starting at P2 by intraperitoneal injection of tamoxifen. Sox10-Fth ${ }^{\mathrm{KO}}\left(\mathrm{Fth}^{\mathrm{f} / \mathrm{f}}\right.$, Sox10-Cre ER $\left.{ }^{\mathrm{Cre} /-}\right)$ and control (Cre negative) littermates ( $\mathrm{Fth}^{\mathrm{f} / \mathrm{f}}$, Sox10-Cre $\mathrm{ER}^{-/-}$) were injected once a day for 5 consecutive days with tamoxifen, and brains were collected at P15 and P30. Postnatal myelination was initially evaluated by immunohistochemistry for myelin proteins. Compared with control littermates, the fluorescent signal for MBP and PLP was reduced in the corpus callosum, cortex, and striatum of Sox10-Fth ${ }^{\mathrm{KO}}$ mice (Fig. 2A,B). This substantial decline in myelin synthesis was more evident at P15 than at P30 (Fig. 2A,B). Additionally, total proteins were collected from the cortex and cerebellum to assess the expression of myelin proteins by Western blot (Fig. 2C,D). In agreement with the immunohistochemistry results, the expression levels of MBP, CNP, and PLP were decreased in Sox10-Fth ${ }^{\mathrm{KO}}$ brains at P15 and P30 (Fig. 2C, D). Again, P15 Sox10-Fth ${ }^{\mathrm{KO}}$ mice showed more significant reductions than P30 animals (Fig. 2C,D). The myelination of the Sox10-Fth ${ }^{\mathrm{KO}}$ CNS was also evaluated using the Black Gold staining technique. In line with the previous results, the corpus callosum and cortex of Sox10-Fth ${ }^{\mathrm{KO}}$ mice showed less myelin than controls (Fig. $3 C, D$ ). To measure intracellular iron quantities, the enhanced Perls histochemistry technique was used. Fewer ironpositive oligodendrocytes with reduced Perls staining intensity were found in Sox10-Fth ${ }^{\mathrm{KO}}$ brains (Fig. $3 A, B$ ). Suggesting an oligodendrocyte-specific iron deficiency, the average Perls staining intensity in cortical neuros and in brain areas, such as the globus pallidus and the substantia nigra, was found to be normal (data not shown).

To further analyze the effect of Fth deletion in oligodendrocyte development, the total number of oligodendrocytes (Olig2-positive cells) and the density of mature myelinating oligodendrocytes 
A

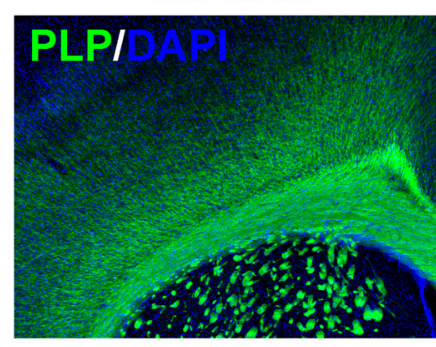

B

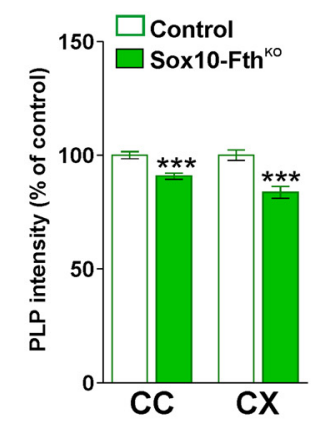

D

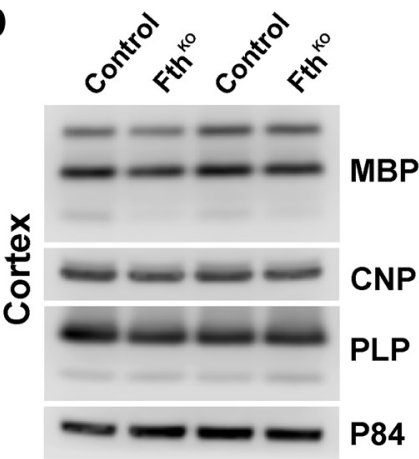

P84
Sox10-Fth

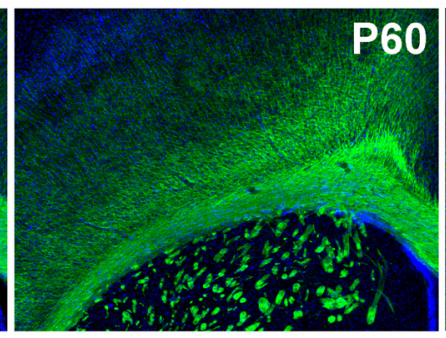

Control

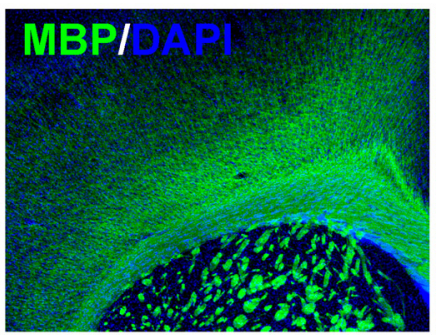

Sox $10-F t^{\text {KO }}$

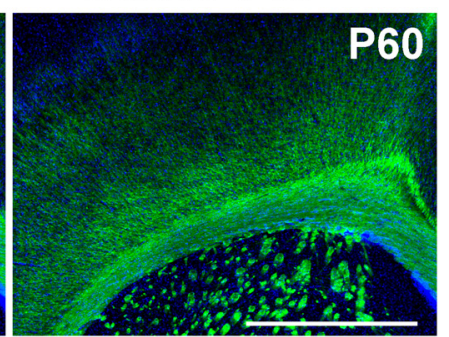

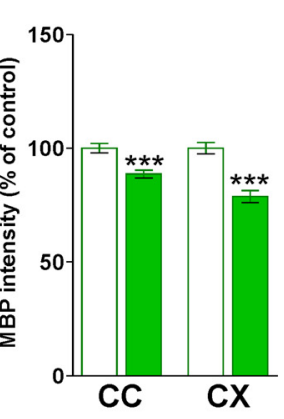

C

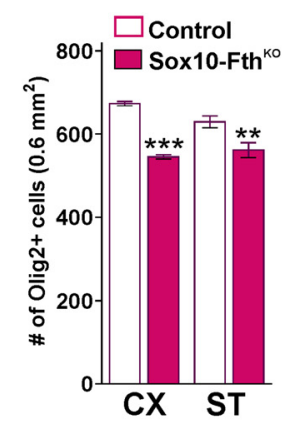

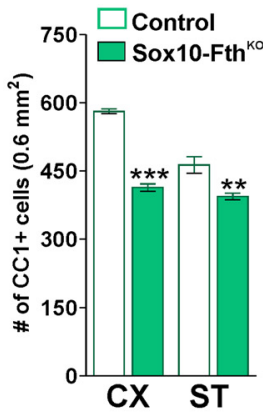

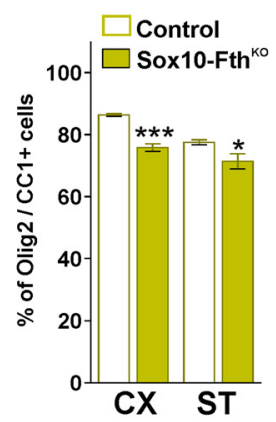

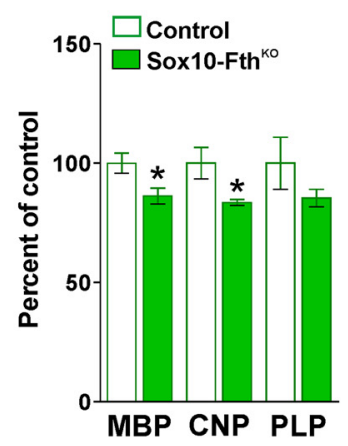
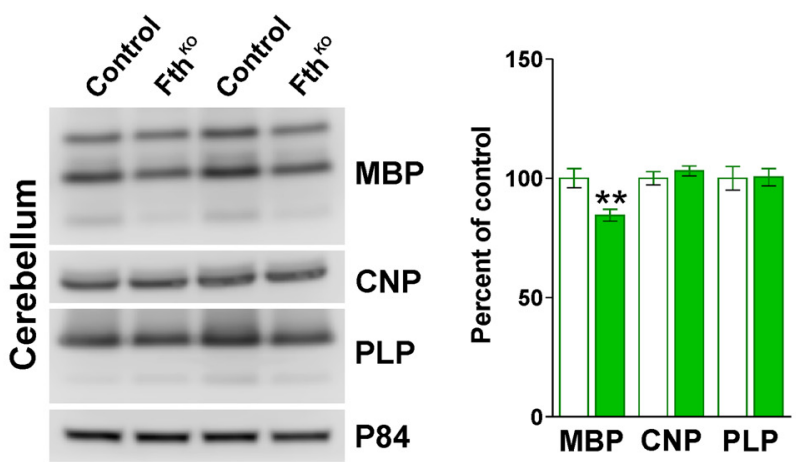

Figure 6. Myelin production and oligodendrocyte maturation in young Sox10-FthKO brains. A, PLP and MBP immunostaining in the brain of control and Sox10-FthK0 mice at P60. Representative brain coronal sections are shown. Scale bar, $180 \mu \mathrm{m} . \boldsymbol{B}, \mathrm{PLP}$ and MBP expression was quantified by analyzing the integrated fluorescence intensity in the central corpus callosum $(C C)$ and in the cortex $(C X)$. C, The total number of Olig2- and CC1-positive cells and the percentage of 0lig2/CC1 double-positive cells were quantified in the cortex (CX) and in the striatum (ST) at P60. Comparisons between experimental groups were made by the unpaired $t$ test. Six brains per experimental condition were analyzed. Values are percentage of control values \pm SEM. ${ }^{*} p<0.05 ;{ }^{* *} p<0.01 ;{ }^{* * *} p<0.001$ versus respective controls. $D$, Total proteins were collected from the cortex and cerebellum at P60 to assess the expression of MBP, CNP, and PLP by Western blot. Representative Western blots are shown. P84 was used as the internal standard. Data from four independent experiments are summarized based on the relative spot intensities and plotted as percent of controls. Values are mean \pm SEM. ${ }^{*} p<0.05 ;{ }^{* *} p<0.01$ versus respective controls.

(CC1-positive cells) were analyzed in the corpus callosum, cortex, and striatum at P15 and P30 (Fig. 4). A substantial reduction in the total number of Olig2-positive cells was observed in all brain areas (Fig. $4 A, B$ ). For instance, the corpus callosum of Sox $10-\mathrm{Fth}^{\mathrm{KO}}$ mice presented an $\sim 60 \%$ decline in the number of Olig2-expressing cells at P15 (Fig. 4B). Higher reductions were found in the CC1-positive oligodendrocyte population (Fig. $4 A, B$ ). The density of these cells was reduced $>80 \%$ in all brain structures at P15, and was diminished $40 \%$ in the cortex and $20 \%$ in the striatum at P30 (Fig. $4 B$ ). The percentage of Olig2/CC1 double-positive cells followed the same trend, with reductions $>50 \%$ at $\mathrm{P} 15$ and declines of $\sim 15 \%-20 \%$ at $\mathrm{P} 30$ (Fig. 4B). Furthermore, oligodendrocyte proliferation was estimated using the mitotic marker Ki67 in combination with Olig2, and the proportion of newly generated OPCs was calculated mixing Olig2 with the transcription factor Sox2 (Fig. 5). Sox10-Fth ${ }^{\mathrm{KO}}$ brains displayed a significant decrease in the percentage of Olig2/Ki67 and Olig2/Sox2 double-positive cells at P15 as well as at P30 (Fig. 5A-C). These changes were evident in the corpus callosum and the subventricular zone and were more pronounced at P15 than at P30 (Fig. 5A-C). Finally, the apoptotic maker Caspase-3 was combined with Olig2 to evaluate oligodendrocyte cell death. The number of Olig2/Caspase-3 double-positive cells was equal among genotypes, suggesting that Fth deletion does not induce apoptotic cell death in vivo (Fig. 5D).

The hypomyelination observed in young Sox10-Fth ${ }^{\mathrm{KO}}$ brains treated with tamoxifen during the first postnatal week was also found at P60 (Fig. 6). Although the fluorescent signal for PLP and $\mathrm{MBP}$ was reduced no more than $15 \%$, these changes were detected in the corpus callosum as well as in the cortex of Sox10Fth ${ }^{\mathrm{KO}}$ mice (Fig. 6A,B,D). Additionally, P60 Sox10-Fth ${ }^{\mathrm{KO}}$ brains showed a significant reduction in the total number of Olig2- and CC1-positive oligodendrocytes in the cortex and striatum (Fig. $6 C)$. To explore the role of Fth in oligodendroglial cells after the peak of myelination, immunohistochemical and Western blot experiments for myelin proteins were performed in adult mice. For this set of experiments, Sox10-Fth ${ }^{\mathrm{KO}}$ mice were injected with tamoxifen at P60 and brain tissue was collected at P90. Compared with control animals, P90 Sox10-Fth ${ }^{\mathrm{KO}}$ mice displayed normal levels of myelin proteins (Fig. $7 A, B, D$ ), and 
A

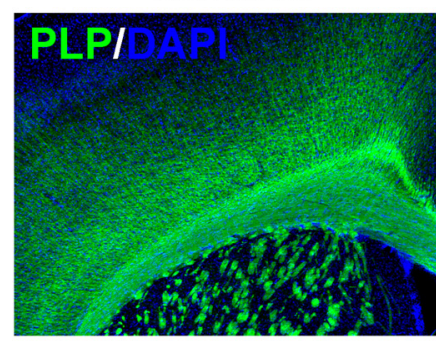

B

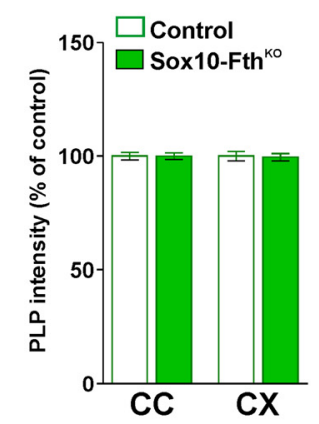

D

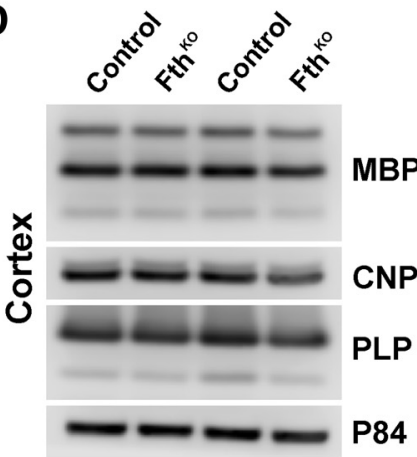

Sox10-Fth

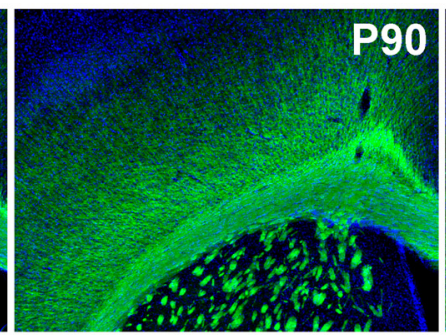

C

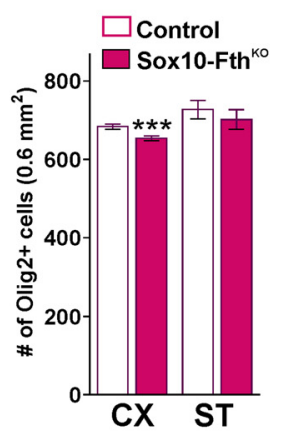

Control

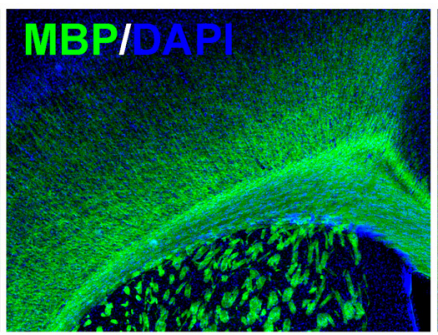

Sox $10-F t^{\mathrm{KO}}$

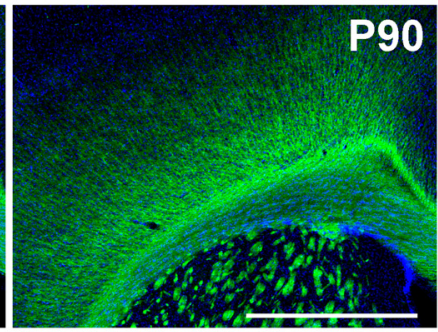

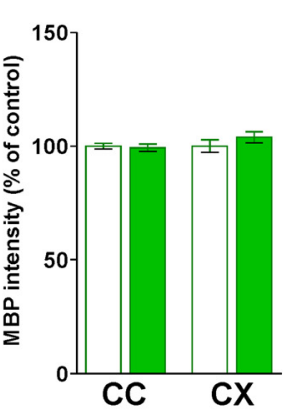

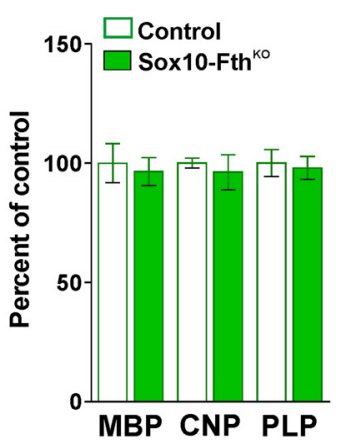

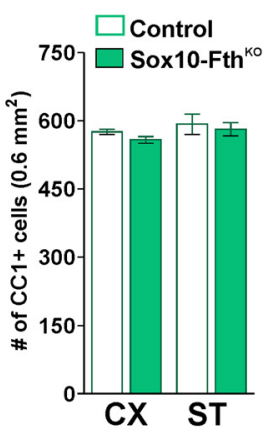

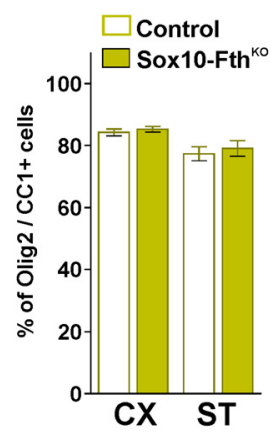

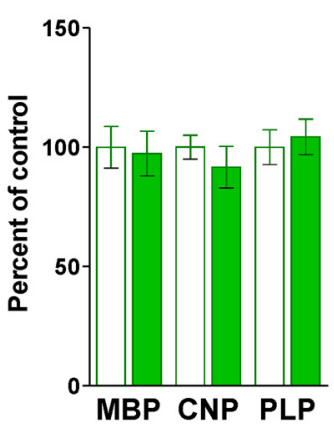

Figure 7. Myelin production and oligodendrocyte numbers in adult Sox10-FthK0 mice. A, PLP and MBP immunostaining in the brain of control and Sox10-FthK0 mice at P90. Representative brain coronal sections are shown. Scale bar, $180 \mu \mathrm{m} . \boldsymbol{B}, \mathrm{PLP}$ and MBP expression was quantified by analyzing the integrated fluorescence intensity in the central corpus callosum $(C C)$ and in the cortex $(C X)$. C, The total number of Olig2- and CC1-positive cells and the percentage of 0lig2/CC1 double-positive cells were quantified in the cortex (CX) and in the striatum (ST) at P90. Comparisons between experimental groups were made by the unpaired $t$ test. Six brains per experimental condition were analyzed. Values are percentage of control values \pm SEM. ${ }^{* * *} p<0.001$ versus respective controls. $\boldsymbol{D}$, Total proteins were collected from the cortex and cerebellum at P90 to assess the expression of MBP, CNP, and PLP by Western blot. Representative Western blots are shown. P84 was used as the internal standard. Data from four independent experiments are summarized based on the relative spot intensities and plotted as percent of controls. Values are mean \pm SEM.

normal numbers of CC1-positive oligodendrocytes in all brain areas (Fig. 7C). However, we detected a reduction in the density of cortical Olig2-expressing cells (Fig. 7C).

The myelination of the Sox10-Fth ${ }^{\mathrm{KO}}$ brain was also assessed by electron microscopy. The degree of myelination was analyzed by calculating the percentage of myelinated axons and the $g$-ratio of myelinated axons in the body of the corpus callosum (Fig. 8). The Sox 10-Fth ${ }^{\mathrm{KO}}$ animals showed an important reduction in the percentage of myelinated axons with changes in the mean $g$-ratio of myelinated axons at both P15 and P30 (Fig. 8A,C,E). At P30, the changes in the $g$-ratio were small; but at P15, the $g$-ratio of low-diameter axons was drastically increased (Fig. 8A-C). Importantly, the average diameter of myelinated axons was augmented in the corpus callosum of Sox10-Fth ${ }^{\mathrm{KO}}$ mice at P15 (Fig. $8 D$ ). In agreement with the immunohistochemical and Western blot data, $\mathrm{P} 90$ brains from Sox10-Fth ${ }^{\mathrm{KO}}$ animals injected with tamoxifen at P60 displayed minor changes in the $g$-ratio as well as in the percentage of myelinated axons (Fig. $8 A-C, E$ ).

Similar experiments were conducted in a conditional $\mathrm{KO}$ mouse in which Fth was ablated in NG2-positive OPCs. NG2-
$\mathrm{Fth}^{\mathrm{KO}}\left(\mathrm{Fth}^{\mathrm{f} / \mathrm{f}}, \mathrm{NG} 2-\mathrm{CreER} \mathrm{Ere}^{\mathrm{Cr}-}\right)$ mice and control (Cre negative) littermates $\left(\mathrm{Fth}^{\mathrm{f} / \mathrm{f}}, \mathrm{NG} 2-\mathrm{CreER}{ }^{-/-}\right)$were injected with tamoxifen during the first postnatal week (P2-P6), and brains were collected at P15 and P30 for analysis. Initially, MBP and PLP expression was evaluated in the corpus callosum, cortex, and striatum by immunohistochemistry. Compared with controls, NG2-Fth ${ }^{\mathrm{KO}}$ mice presented a significant decrease in MBP and PLP immunostaining in all brain areas analyzed (Fig. $9 A, B$ ). Similar to what we found in Sox10-Fth ${ }^{\mathrm{KO}}$ mice, NG2-Fth ${ }^{\mathrm{KO}}$ animals showed a more prominent reduction in myelin protein expression at $\mathrm{P} 15$ than at P30 (Fig. 9A,B). Furthermore, examination of the oligodendrocyte population reveals a substantial decrease in the density of CC1-expressing cells and a decline in the fraction of Olig2/CC1-positive cells (Fig. 9C,D).

To define the temporal window in which oligodendrocytes incorporate and store iron, Sox10-Fth ${ }^{\mathrm{KO}}$ mice were treated with tamoxifen during the third postnatal week (P15 to P19) and brains were then collected for examination at P30 and P60 (Fig. 10). We detected reductions in the expression of myelin proteins (MBP and PLP), in the density of total oligodendrocytes (Olig2- 

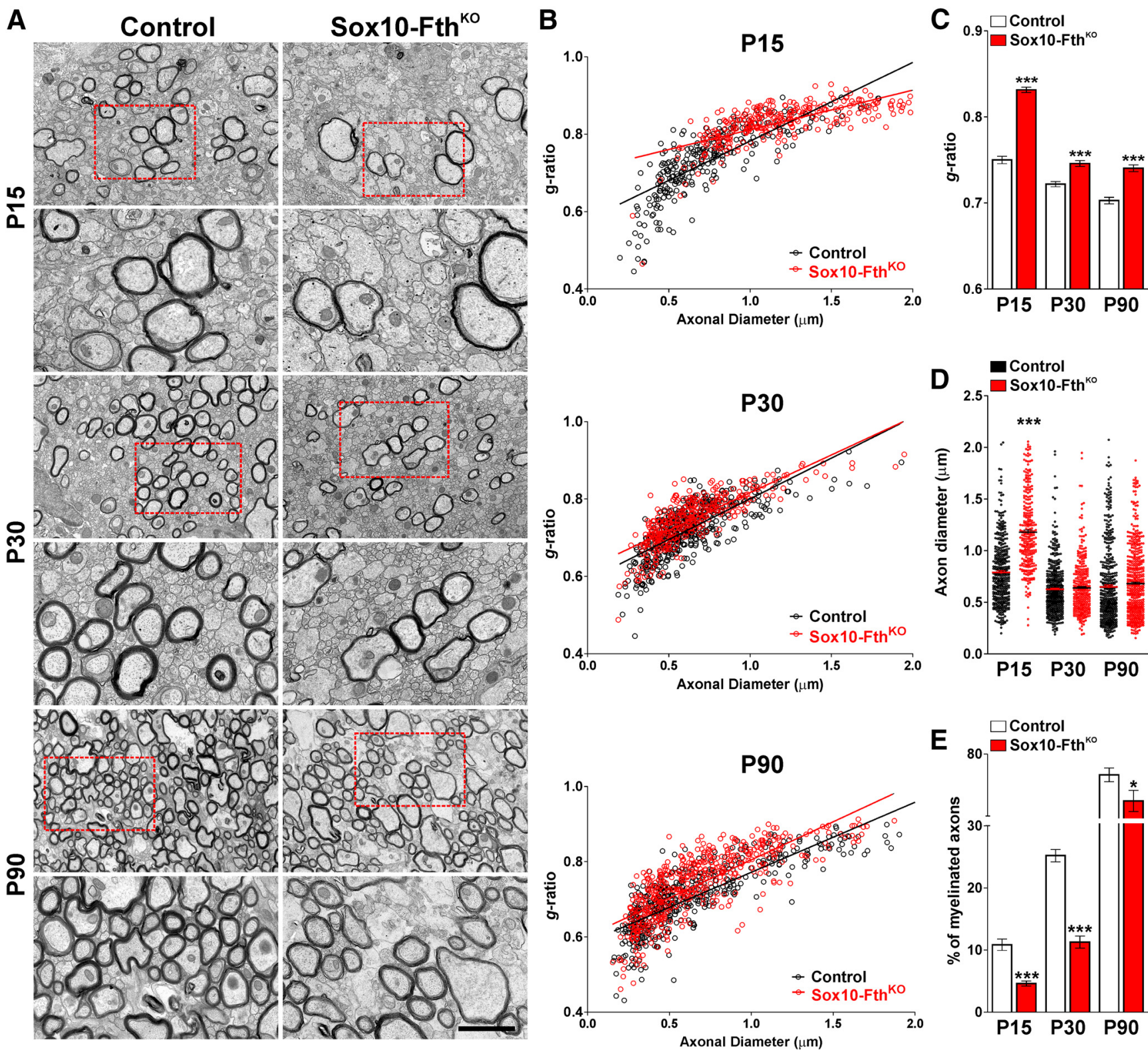

Figure 8. Electron microscopy of the Sox10-FthKO corpus callosum. $A$, Electron micrographs of axons in the body of the corpus callosum of control and Sox10-FthK0 mice at P15, P30, and P90. Scale bars: Top, $8 \mu \mathrm{m}$; Bottom, $2 \mu \mathrm{m}$. B, Scatter plot of $g$-ratio values of myelinated axons. C, Mean $g$-ratio values of myelinated axons for the same experimental conditions. $\boldsymbol{D}$, Mean axonal diameter of myelinated axons. $\boldsymbol{E}$, Percentage of myelinated axons. Four animals per experimental group and 150 fibers were analyzed. Values are mean \pm SEM. ${ }^{*} p<0.05 ;{ }^{* * *} p<0.001$ versus control.

positive cells), and in the number of CC1-expressing cells (Fig. $10 A-D)$. These changes were found in several Sox $10-\mathrm{Fth}^{\mathrm{KO}}$ brain structures and were larger at P30 than at P60 (Fig. 10A-D). For example, at $\mathrm{P} 30$, the average reduction in $\mathrm{MBP}$ immunostaining in the lateral corpus callosum and in the cortex was higher than $50 \%$, and the decline in the number of Olig2- and CC1-positive cells in the cortex was larger than 20\% (Fig. 10A-D). These changes were similar in magnitude to the ones found in Sox10$\mathrm{Fth}^{\mathrm{KO}}$ animals in which Fth was knocked down in oligodendroglial cells at P2. These data suggest that the temporal window in which oligodendroglial cells uptake and store iron in Fth extends beyond the third postnatal week.

The role of Fth in the remyelination of the adult brain To determine how the loss of Fth affects the remyelination potential of OPCs in the adult brain, we used the CPZ model of demyelination and remyelination. P60 control $\left(\mathrm{Fth}^{\mathrm{f} / \mathrm{f}}, \mathrm{NG} 2-\right.$ CreER $\left.{ }^{-1-}\right)$ and NG2-Fth ${ }^{\mathrm{KO}}\left(\mathrm{Fth}^{\mathrm{f} / \mathrm{f}}, \mathrm{NG} 2-\mathrm{CreER}{ }^{\mathrm{Cre} /-}\right)$ mice were treated with CPZ for 7 weeks to induce demyelination and were injected with tamoxifen every other day during the last 2 weeks of the CPZ diet to delete the Fth gene specifically in NG2-positive OPCs. Brains were then collected for analysis after 7 weeks of $\mathrm{CPZ}$ treatment $(\mathrm{CPZ})$ and at 2 or 4 weeks of recovery in normal diet (2W or $4 \mathrm{~W}$ Rec). Additionally, brain tissue was collected from mice that were not treated with $\mathrm{CPZ}$ or tamoxifen (Untreated). Seven weeks of CPZ treatment induces a severe demyelination in the brain of both controls and NG2- $\mathrm{Fth}^{\mathrm{KO}}$ mice (Fig. 11A,B). Importantly, at this time point, we found a further decrease of MBP and PLP expression in the cortex of NG2$\mathrm{Fth}^{\mathrm{KO}}$ mice (Fig. 11A,B). A significant recovery in MBP and PLP levels was observed in the corpus callosum and cortex of both genotypes during the remyelination phase of the $\mathrm{CPZ}$ model; but 

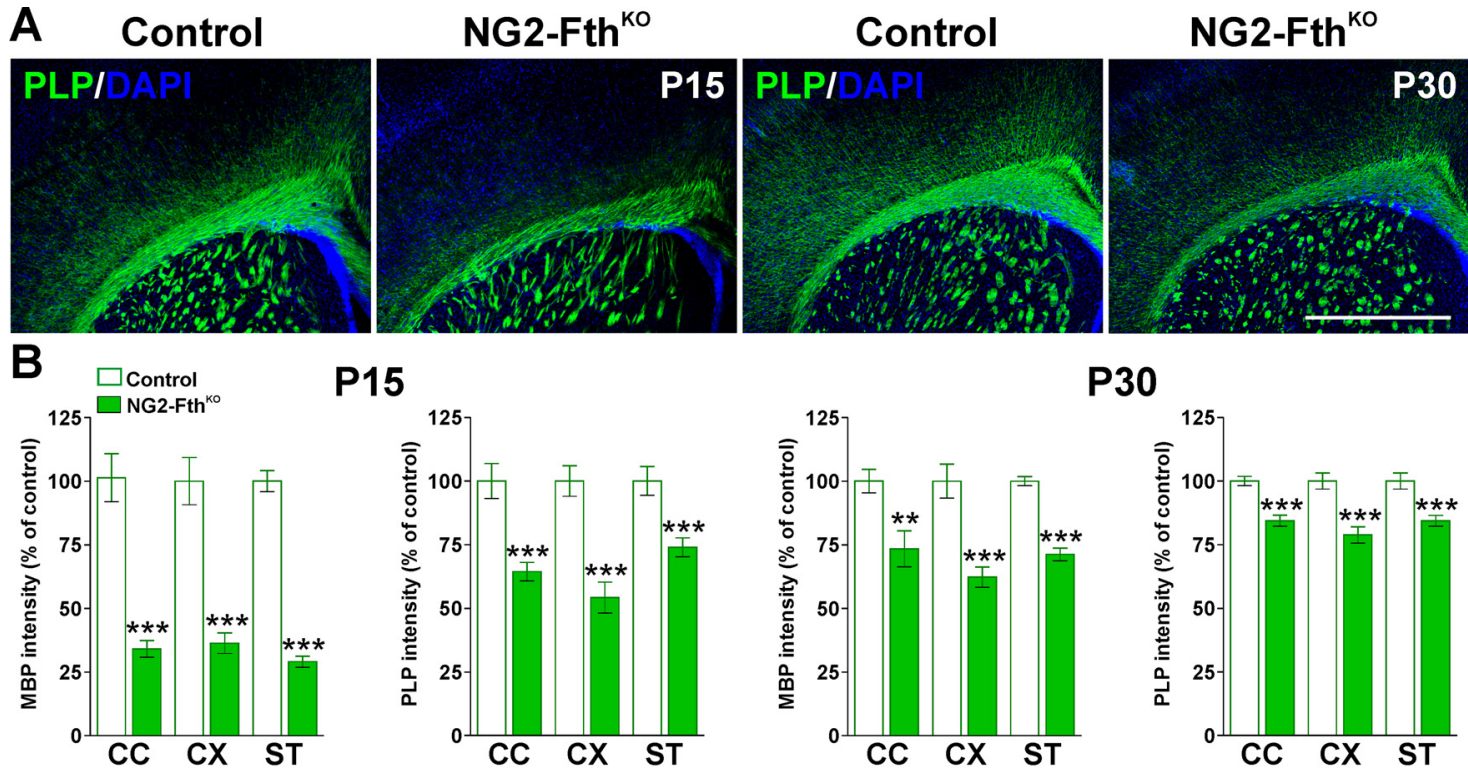

P15
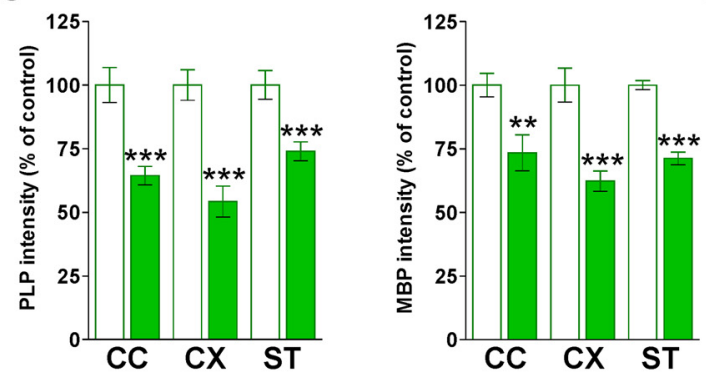

P30

C

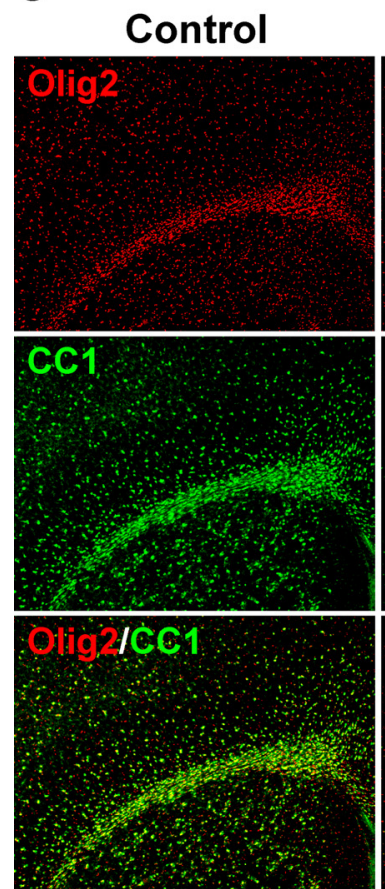

NG2-Fth ${ }^{\mathrm{KO}}$
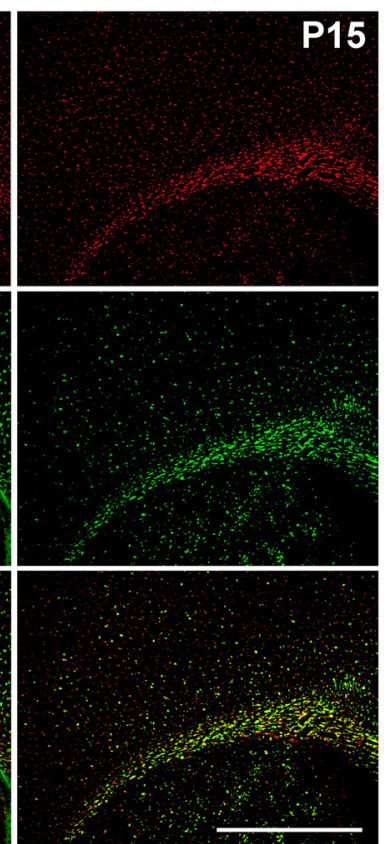

D

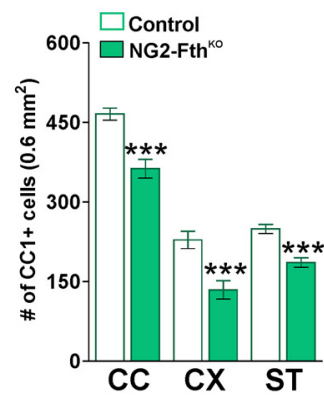

\section{P15}
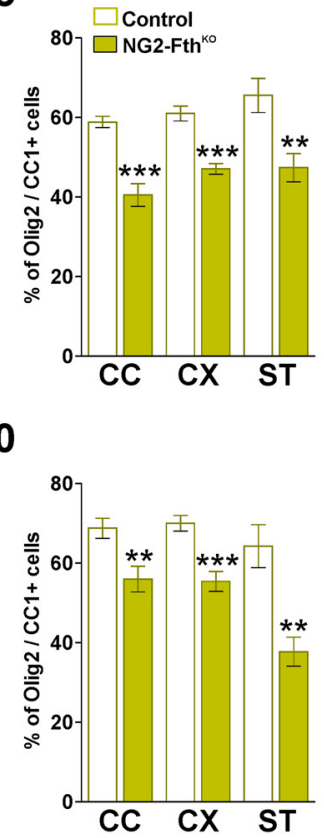

Figure 9. Myelination and oligodendrocyte numbers in the postnatal NG2-FthKO brain. A, PLP immunostaining in the brain of control and NG2-FthKO mice at P15 and P30. Representative brain coronal sections are shown. Scale bar, $180 \mu \mathrm{m} . \boldsymbol{B}$, MBP and PLP expression was quantified by analyzing the integrated fluorescence intensity in the central corpus callosum (CC), in the cortex (CX), and in the striatum (ST) at P15 and P30. C, Representative coronal sections from control and NG2-FthK0 brains at P15 immunostained for 0lig2 and CC1. Scale bar, $180 \mu \mathrm{m}$. D, The total number of CC1-positive cells and the percentage of 0lig2/CC1 double-positive cells were quantified in the central area of the corpus callosum (CC), in the cortex (CX), and in the striatum (ST) at P15 and P30. Comparisons between experimental groups were made by the unpaired $t$ test. Six brains per experimental condition were analyzed. Values are percentage of control values \pm SEM. ${ }^{* *} p<0.01 ;{ }^{* * *} p<0.001$ versus respective controls.

suggesting a deficit in remyelination, NG2-Fth ${ }^{\mathrm{KO}}$ mice showed significantly less MBP and PLP production than controls after 2 and 4 weeks of recovery (Fig. $11 A, B$ ). These reductions were also found in Western blot experiments for myelin proteins (Fig. $11 C)$. We found no differences in the number of Olig2-positive cells among genotypes in any of the experimental conditions (Fig. 11B). However, we detected a decrease in the number of CC1-positive cells at the end of the CPZ treatment and after 2 and 4 weeks of recovery in Fth-deficient mice (Fig. 11B). Remyelination was also evaluated by EM at the body of corpus callosum by determining the $g$-ratio and the percent of myelinated axons. No relevant changes in the average diameter of myelinated axons were found (Fig. 12A,B). However, the corpus callosum of $\mathrm{NG} 2-\mathrm{Fth}^{\mathrm{KO}}$ animals presented a significant increase in the average $g$-ratio (Fig. $12 A, C, E$ ) and a clear reduction in the percent of myelinated axons at both 7 weeks of CPZ treatment and 2 weeks of recovery (Fig. 12A,D).

\section{Discussion}

Iron deficiency in late-prenatal and early-postnatal periods can lead to long-term neurobehavioral deficits in humans, including impairments in learning and memory (Lozoff, 2007; Radlowski 
A Control
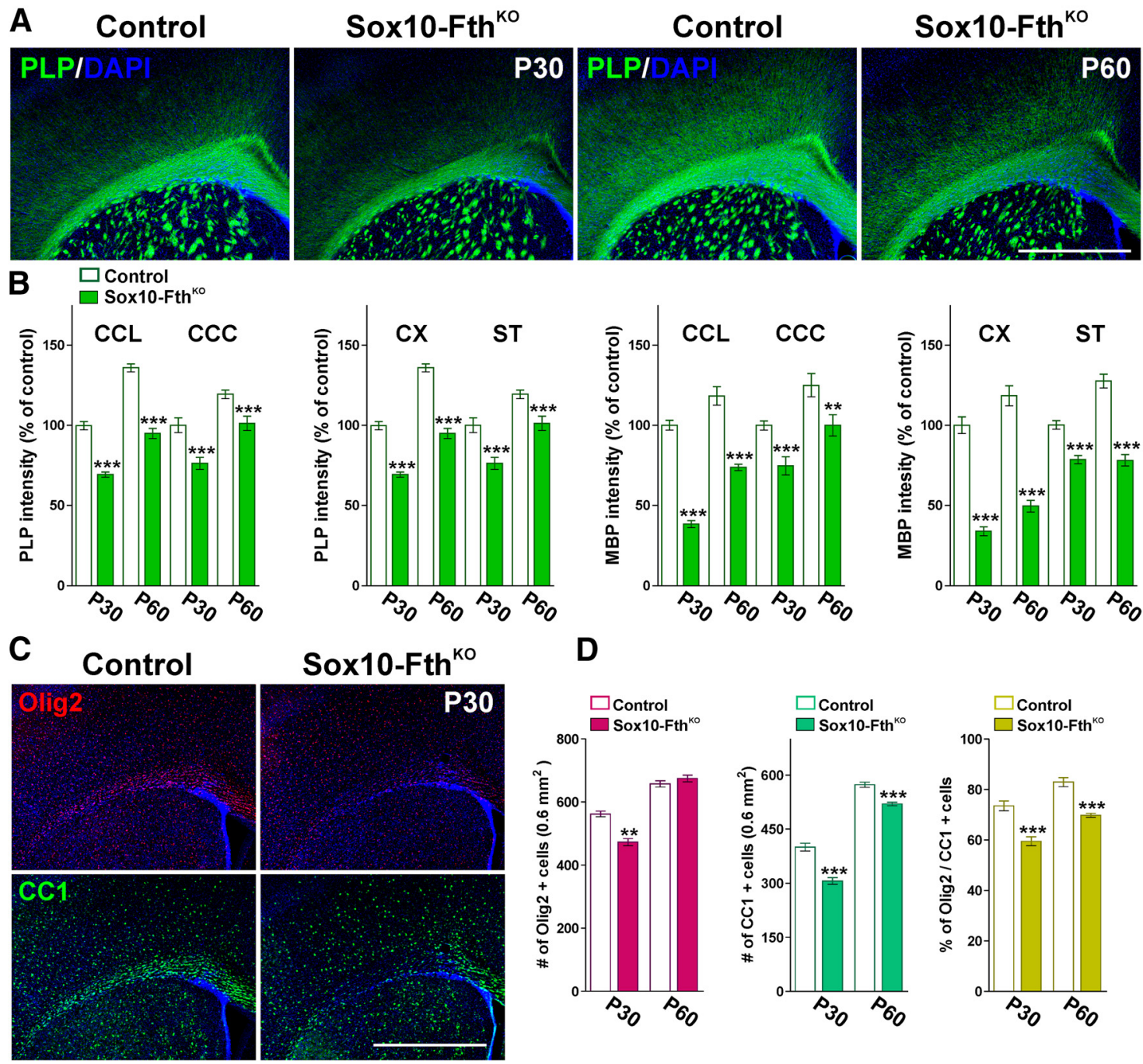

D
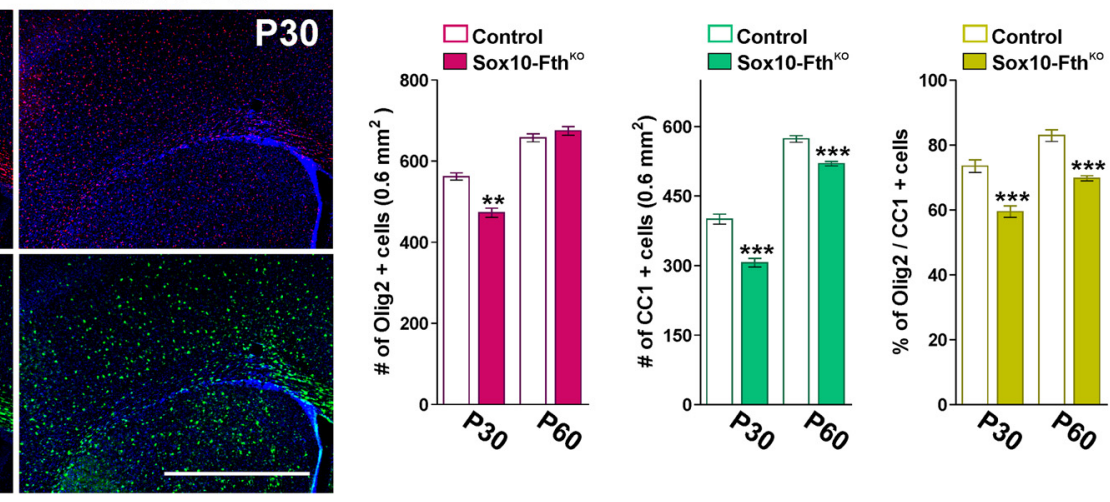

Figure 10. Myelin synthesis and oligodendrocyte quantities in young Sox10-FthK0 mice. $\boldsymbol{A}, \mathrm{PLP}$ immunostaining in the brain of control and Sox10-FthK0 mice at P30 and P60. Representative brain coronal sections are shown. Scale bar, $180 \mu \mathrm{m}$. B, PLP and MBP expression was quantified by analyzing the integrated fluorescence intensity in the lateral (CCL) and central corpus callosum (CCC), in the cortex (CX) and in the striatum (ST). C, Representative coronal sections from control and Sox10-FthK0 brains at P30 immunostained for 0lig2 and CC1. Scale bar, $180 \mu \mathrm{m}$. D , The total number of 0lig2- and CC1-positive cells and the percentage of 0lig2/CC1 double-positive cells were quantified in the cortex at P30 and P60. Comparisons between experimental groups were made by the unpaired $t$ test. Six brains per experimental condition were analyzed. Values are percentage of control values \pm SEM. ${ }^{* *} p<0.01 ;{ }^{* * *} p<0.001$ versus respective controls.

and Johnson, 2013). The neurocognitive problems of iron deficiency during this critical perinatal time frame are difficult to remedy, persisting into adulthood. Thus, understanding how the different cell types of the CNS incorporate and manage iron through maturity is critical to develop new means to amend cognitive disfunctions in individuals who experienced early iron deficiency. Until now, our knowledge regarding brain iron metabolism was generated from animal models of dietary iron restriction, or mutants and full $\mathrm{KO}$ animals for iron proteins. In contrast, the conditional $\mathrm{KO}$ mice described in this work allowed us to study the consequences of iron deficit specifically in the oligodendrocyte lineage without the interference of broad brain iron deficiency.

\section{The importance of iron storage in oligodendrocyte} development and myelination

Oligodendrocytes require iron as a cofactor for several enzymes involved in the proliferation and differentiation of OPCs, and in the production of cholesterol and phospholipids, which are essential myelin components (Stephenson et al., 2014). OPCs as well as mature myelinating oligodendrocytes are the highest iron-rich cells in the brain (Connor and Menzies, 1996). It was recently established that the average intracellular iron concentration in cortical oligodendrocytes $(3.05 \mathrm{~mm})$ doubles the iron content of astrocytes and microglia cells and quintuples the average in neurons (Reinert et al., 2019). Assuming a load of 2400 atoms of iron per ferritin molecule (P. Zhang et al., 2005), rat oligodendrocytes contain $\sim 690$ ferritin molecules per $\mu \mathrm{m}^{3}$ versus 130 in cortical neurons (Reinert et al., 2019). In this work, we have found that Fth ablation during early brain development in Sox10- or NG2-positive oligodendroglial cells significantly delays the myelination of several brain structures. The corpus callosum of $\mathrm{Fth}^{\mathrm{KO}}$ animals presented a significant decrease in the thickness of myelin sheaths and in the percentage of myelinated axons. Sox10-Fth ${ }^{\mathrm{KO}}$ brains displayed a widespread decline in the number of iron-positive oligodendrocytes as well as a substantial decrease in the average Perls staining intensity per cell. Although a requirement of iron for myelin production is generally accepted, it is not clear whether iron deficiency leads to global brain hypomyelination by affecting oligodendrocyte numbers or 
A

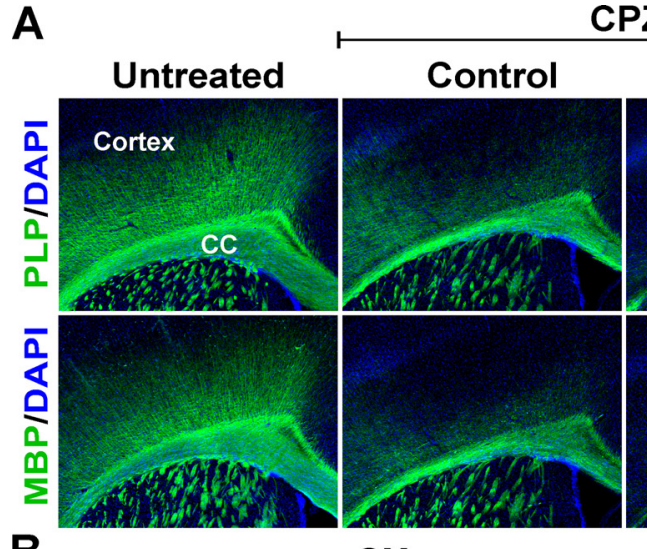

CPZ

2W Rec

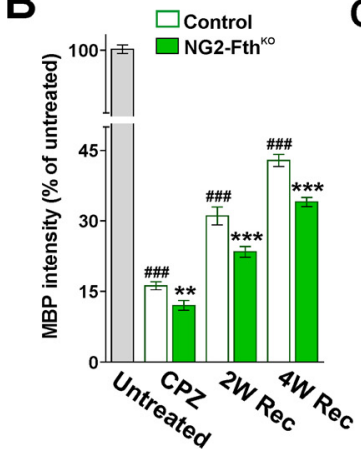

CX
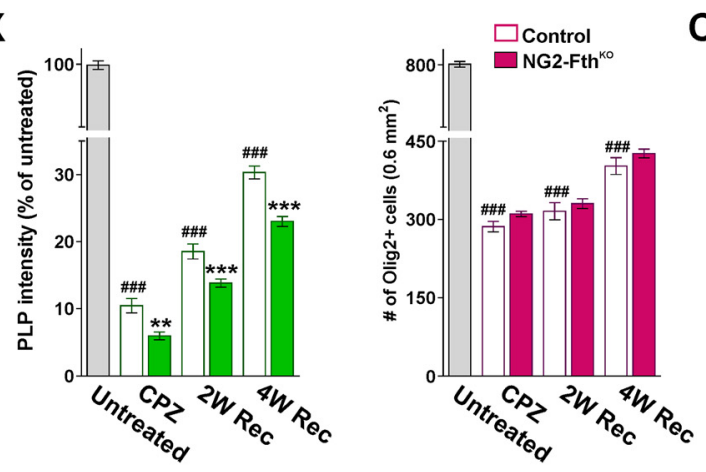

CX

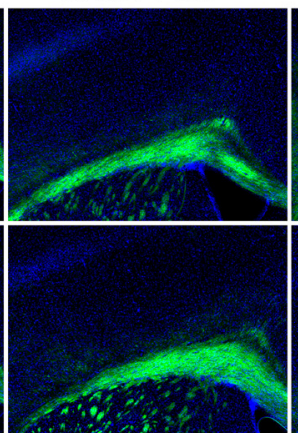

\section{Control}

NG2-Fth ${ }^{\text {Ko }}$

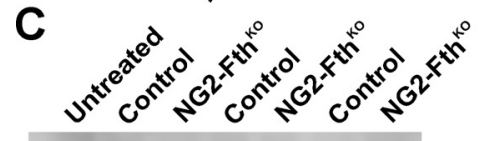

MBP

MOG

PLP
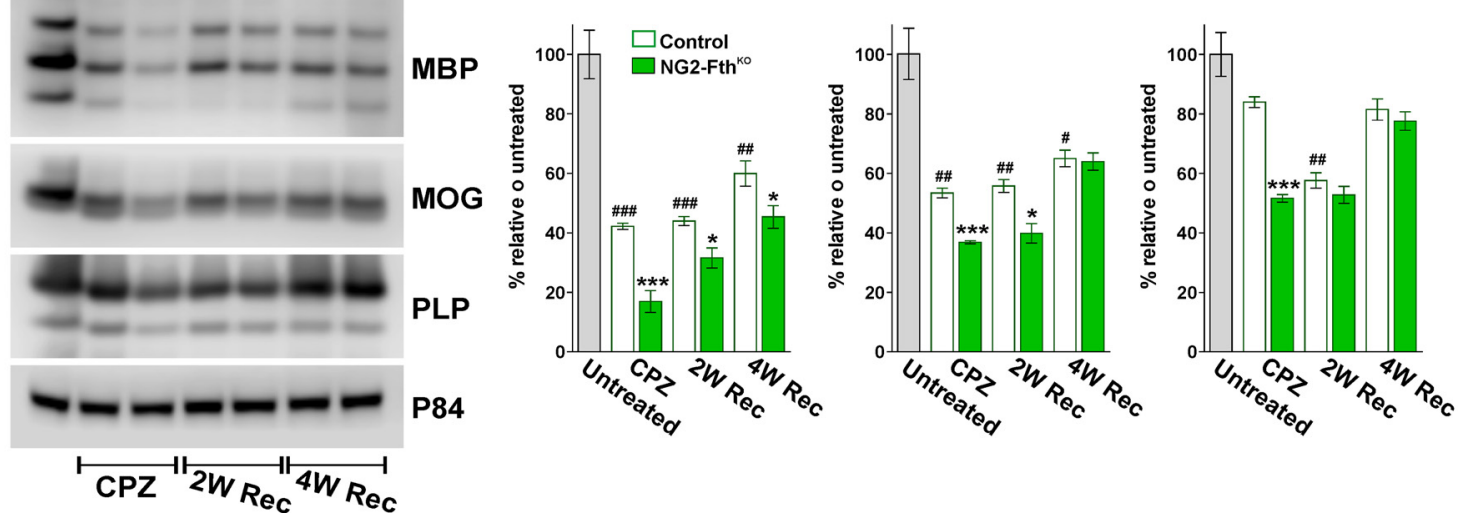

Figure 11. Remyelination in the adult NG2-FthKO brain. $A$, Representative coronal sections from control and NG2-FthKO brains immunostained for PLP and MBP. Tissue was collected from untreated, control, and NG2-FthKO mice at the end of the (PZ treatment and after 2 weeks of recovery (2W Rec). Scale bar, $180 \mu \mathrm{m} . \boldsymbol{B}$, MBP and PLP fluorescent intensity, and total numbers of Olig2- and CC1-positive cells were quantified in the cortex (CX) of untreated, control, and NG2-FthKO mice at the end of the CPZ treatment and after 2 and 4 weeks of recovery (2W or 4W

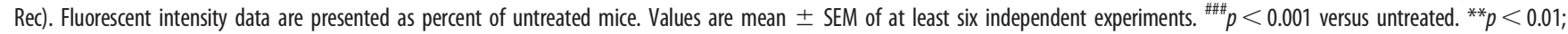
${ }^{* * *} p<0.001$ versus control. C, Representative Western blots for MBP, MOG, and PLP made with brain tissue comprised of the corpus callosum and cortex. p84 was used as the internal standard. Data from four independent experiments are summarized based on the relative spot intensities and plotted as percent of untreated mice. Values are mean \pm SEM. ${ }^{\#} p<0.05$; ${ }^{\#} p<0.01$; $\#^{\# \#}<0.001$ versus untreated. ${ }^{*} p<0.05 ;{ }^{* * *} p<0.001$ versus control.

their differentiation state or both (Todorich et al., 2009). We have found a reduction in the population of oligodendrocytes as well as in the numbers of proliferating OPCs across the brain of $\mathrm{Fth}^{\mathrm{KO}}$ animals. This suggests that Fth iron storage is important for OPC proliferation and that a decline in OPC quantities could be responsible for the hypomyelination observed in young $\mathrm{Fth}^{\mathrm{KO}}$ animals. However, the proportion of mature oligodendrocytes was reduced in all brain areas of the Sox10 and NG2-Fth ${ }^{\mathrm{KO}}$ mice, suggesting that the rate of OPC differentiation is also affected in the absence of Fth.

As a result of its ability to sequester iron rapidly, Fth is considered an antioxidant protein (Balla et al., 2007) because iron removed quickly by this protein is not available for inducing oxidative damage. For example, increased cytoplasmic iron levels and ROS formation were found in Fth KO fibroblasts, which significantly reduce the viability of these cells in vitro (Darshan et al., 2009). Fth appears to have a similar protective role in oligodendrocyte physiology. For instance, Fth synthesis increased in oligodendrocytes following exposure to hypoxic conditions or TNF $\alpha$ (Qi and Dawson, 1994; Sanyal and Szuchet, 1995). Thus, the vulnerability of oligodendroglial cells to oxidative damage and the expression of Fth are connected. In the absence of Fth, oligodendrocytes may experience an increase in free cytoplasmic iron, ROS formation, and oxidative stress. We found no changes 

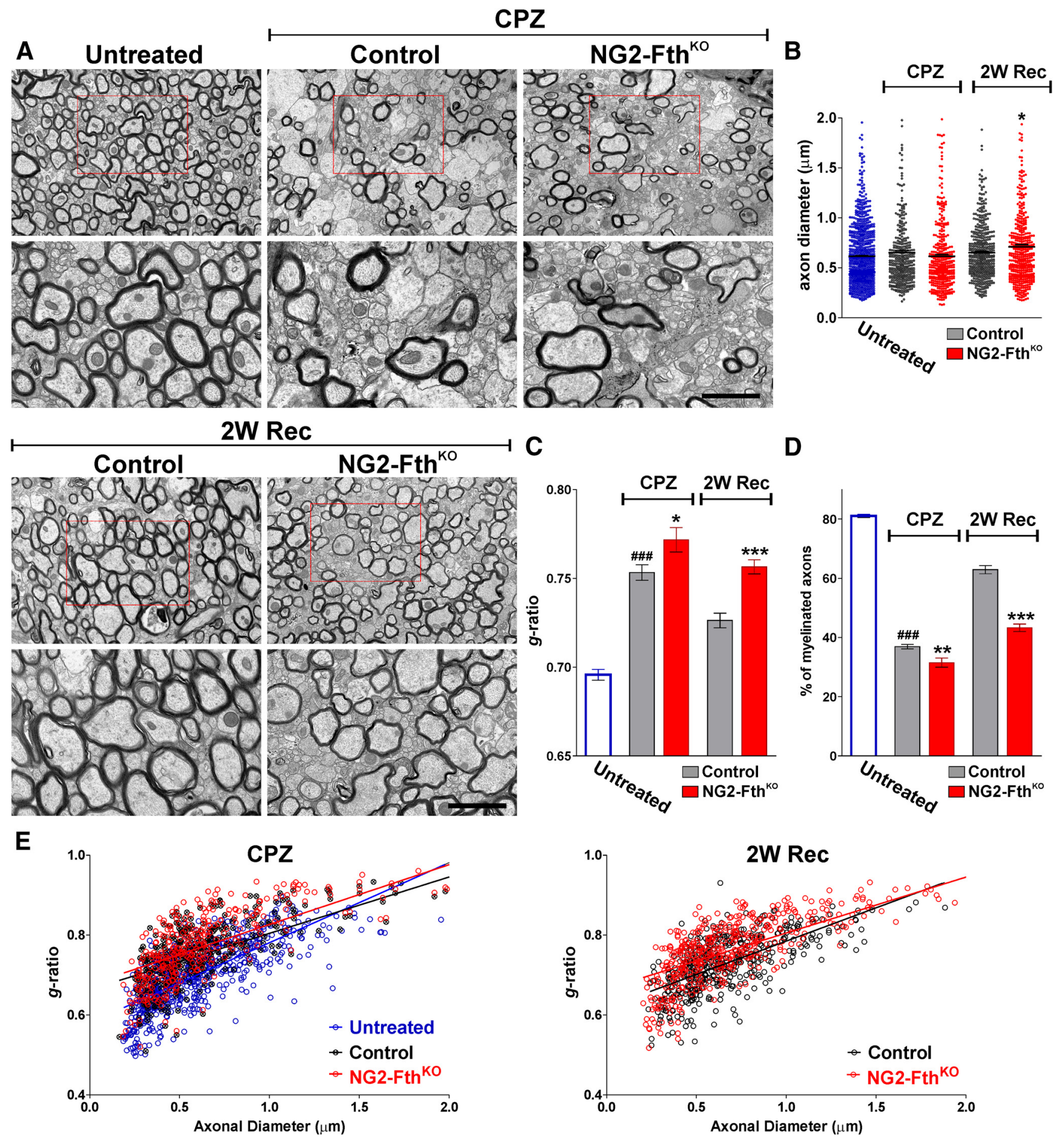

Figure 12. Electron microscopy of the remyelinating NG2-FthKO corpus callosum. $A$, Electron micrographs of axons in the body of the corpus callosum of untreated, control, and NG2-FthKO mice at the end of (PZ treatment and after 2 weeks of recovery (2W Rec). Scale bars: Top, $8 \mu \mathrm{m}$; Bottom, $2 \mu \mathrm{m}$. B, Mean axonal diameter of myelinated axons of untreated, control, and NG2FthKO mice at the end of the (PZ treatment and after 2 weeks of recovery (2W Rec). $C$, Mean $g$-ratio values of myelinated axons for the same experimental conditions. $D$, Percentage of myelinated axons. $\boldsymbol{E}$, Scatter plot of $g$-ratio values. Four animals per experimental group and 150 fibers were analyzed. Values are mean \pm SEM. ${ }^{\# \#} p<0.001$ versus untreated. ${ }^{*} p<0.05$; ${ }^{* *} p<0.01 ;{ }^{* * *} p<0.001$ versus control.

in caspase-3 expression between controls and Fth ${ }^{\mathrm{KO}}$ oligodendrocytes; therefore, losing Fth does not induce oligodendrocyte apoptotic cell death in our mouse model. However, several antioxidant enzymes, such as the glutathione peroxidase, the superoxide dismutase, and the stress-induced heme oxygenase-1, were upregulated in cultured Fth ${ }^{\mathrm{KO}}$ oligodendrocytes. Thus, we cannot entirely exclude the possibility that $\mathrm{Fth}^{\mathrm{KO}}$ oligodendrocytes do not proliferate or mature normally because of excessive ROS formation and oxidative stress. It has been recently established that Fth secreted by myelinating oligodendrocytes protects axons against iron-mediated injury in the adult brain (Mukherjee et al., 2020). Although the Fth secretory pathway is not completely understood, these results suggest that Fth production in mature oligodendrocytes is central for an effective brain antioxidant system. 
In rodents, myelination starts in the first postnatal week, peaks around P30, and is almost complete by P60 (Bercury and Macklin, 2015). The speed of myelin production correlates very well with changes in iron concentrations in several brain regions (Todorich et al., 2009). For example, Tarohda et al. (2004) found rising iron concentrations during early postnatal development from P1 to P40, reaching adult levels by P70. These data suggest that, during the first 2 postnatal months, oligodendroglial cells need to develop all the necessary iron-related mechanisms and regulatory circuits to safely incorporate and accumulate elevated amounts of iron. To define the temporal window in which oligodendroglial cells incorporate and store iron, we have deleted Fth in Sox10-positive cells at three specific developmental time points: P2, P15, and P60. Sox10-Fth ${ }^{\mathrm{KO}}$ mice treated with tamoxifen at the beginning of the first postnatal week (P2), or at the initiation of the third postnatal week (P15), presented a substantial reduction in myelin production and in the density of total and mature oligodendrocytes. In contrast, the ablation of Fth in Sox10-positive cells after P60 has no effect on myelination and/ or oligodendrocyte numbers. These results indicate that, after the completion of the myelination process, new synthesis of Fth is no longer needed for proper oligodendrocyte maturation and/or myelin synthesis. However, since the Sox10 promoter is very active in all oligodendrocyte developmental stages, our experiments do not directly identify the maturational step in which these effects occur.

\section{OPC iron storage in demyelination and remyelination}

Iron is required in many enzymes that influence the metabolism, proliferation, and differentiation of the oligodendrocyte lineage. Therefore, OPC iron deficiency would likely negatively affect the remyelination process (Stephenson et al., 2014). We have found a significant reduction in the remyelination potential of $\mathrm{Fth}^{\mathrm{KO}}$ OPCs. NG2-Fth ${ }^{\mathrm{KO}}$ mice showed a substantial decline in the number of mature oligodendrocytes and myelin synthesis during the remyelination phase of the $\mathrm{CPZ}$ model. Furthermore, thinner myelin sheets and less remyelinated axons were detected in the NG2-Fth ${ }^{\mathrm{KO}}$ corpus callosum after 2 and 4 weeks of recovery. Similar results were found at the end of the $\mathrm{CPZ}$ treatment. After 7 weeks of $\mathrm{CPZ}$ intoxication, NG2-Fth ${ }^{\mathrm{KO}}$ brains presented fewer mature oligodendrocytes and less myelin than controls. These results indicate that NG2-positive OPCs in the adult brain need to synthesize new Fth to normally mature and efficiently remyelinate the CNS. At the same time, these data reveal that adult OPCs need to incorporate and store iron in Fth through the progression of the remyelination process. Therefore, iron availability in the demyelinated brain parenchyma might be a key factor for an efficient remyelination.

Brain iron metabolism has been shown to be disrupted in demyelinated diseases. Abnormal iron deposits were found in both gray and white matter structures in MS patients and animal models of MS (Rouault, 2013). Iron deposits were found to be associated with reactive astrocytes and microglial cells (Ward et al., 2014), and were detected during the active stage of the disease as well as during the recovery phase (Ward et al., 2014). Activated astrocytes and microglia may acquire high levels of iron by phagocytosing myelin and oligodendrocyte debris (Singh and Zamboni, 2009; Williams et al., 2011, 2012). However, the mechanism by which astrocytes and microglial cells release iron back to the brain parenchyma - and if this iron is, to some extent, available to OPCs during remyelination - is completely unknown. Interestingly, high levels of apo-transferrin in the brain of demyelinated rats significantly promote remyelination and OPC differentiation (Adamo et al., 2006). In the same line, Schulz et al. (2012) found that OPC differentiation and remyelination were impaired in a mouse model of focal chemical demyelination, when ferroportin, a transmembrane iron exporter, was deleted specifically in astrocytes. Jointly, these data and our results highlight the importance of iron availability in the demyelinated mouse brain and suggest that iron levels and/or iron redistribution during demyelination can significantly influence the effectiveness of the remyelination process.

\section{References}

Adamo AM, Paez PM, Escobar Cabrera OE, Wolfson M, Franco PG, Pasquini JM, Soto EF (2006) Remyelination after cuprizone-induced demyelination in the rat is stimulated by apotransferrin. Exp Neurol 198:519-529.

Arosio P, Ingrassia R, Cavadini P (2009) Ferritins: a family of molecules for iron storage, antioxidation and more. Biochim Biophys Acta 1790:589599.

Badaracco ME, Ortiz EH, Soto EF, Connor JR, Pasquini JM (2008) Effect of transferrin on hypomyelination induced by iron deficiency. J Neurosci Res 86:2663-2673.

Balla J, Vercellotti GM, Jeney V, Yachie A, Varga Z, Jacob HS, Eaton JW, Balla G (2007) Heme, heme oxygenase, and ferritin: how the vascular endothelium survives (and dies) in an iron-rich environment. Antioxid Redox Signal 9:2119-2137.

Beard JL, Wiesinger JA, Connor JR (2003) Pre- and postweaning iron deficiency alters myelination in Sprague-Dawley rats. Dev Neurosci 25:308315.

Bercury K, Macklin WB (2015) Dynamics and mechanisms of CNS myelination. Dev Cell 32:447-458.

Bou-Abdallah F (2010) The iron redox and hydrolysis chemistry of the ferritins. Biochim Biophys Acta 1800:719-731.

Cheli VT, Santiago-González DA, Spreuer V, Paez PM (2015) Voltage-gated $\mathrm{Ca}^{++}$entry promotes oligodendrocyte progenitor cells maturation and myelination in vitro. Exp Neurol 265:69-83.

Cheli VT, Santiago-González DA, Namgyal LT, Spreuer V, Handley V, Murphy GG, Paez PM (2016) Conditional deletion of the L-type calcium channel Cav1.2 in oligodendrocyte progenitor cells affects postnatal myelination in mice. J Neurosci 36:10853-10869.

Cheli VT, Santiago-González DA, Marziali LN, Zamora NN, Guitart ME, Spreuer V, Pasquini JM, Paez PM (2018) The divalent metal transporter 1 (DMT1) is required for iron uptake and normal development of oligodendrocyte progenitor cells. J Neurosci 38:9142-9159.

Connor JR, Menzies SL (1996) Relationship of iron to oligodendrocytes and myelination. Glia 17:83-93.

Darshan D, Vanoaica L, Richman L, Beermann F, Kühn LC (2009) Conditional deletion of ferritin $\mathrm{H}$ in mice induces loss of iron storage and liver damage. Hepatology 50:852-860.

Franklin KB, Paxinos G (2008) The mouse brain in stereotaxic coordinates. San Diego: Academic.

LeVine SM, Macklin WB (1990) Iron-enriched oligodendrocytes: a reexamination of their spatial distribution. J Neurosci Res 26:508-512.

Li Y, Guan Q, Chen Y, Han H, Liu W, Nie Z (2013) Transferrin receptor and ferritin- $\mathrm{H}$ are developmentally regulated in oligodendrocyte lineage cells. Neural Regen Res 8:6-12.

Lozoff B (2007) Iron deficiency and child development. Food Nutr Bull 28: S560-S571.

Matsuoka T, Ahlberg PE, Kessaris N, Iannarelli P, Dennehy U, Richardson WD, McMahon AP, Koentges G (2005) Neural crest origins of the neck and shoulder. Nature 436:347-355.

Mukherjee C, Kling T, Russo B, Miebach K, Kess E, Schifferer M, Pedro LD, Weikert U, Fard MK, Kannaiyan N, Rossner M, Aicher ML, Goebbels S, Nave KA, Krämer-Albers EM, Schneider A, Mikael Simons M (2020) Oligodendrocytes provide antioxidant defense function for neurons by secreting ferritin heavy chain. Cell Metab 32:259-272.

Ortiz E, Pasquini JM, Thompson K, Felt B, Butkus G, Beard J, Connor JR (2004) Effect of manipulation of iron storage, transport, or availability on myelin composition and brain iron content in three different animal models. J Neurosci Res 77:681-689. 
Qi Y, Dawson G (1994) Hypoxia specifically and reversibly induces the synthesis of ferritin in oligodendrocytes and human oligodendrogliomas. J Neurochem 63:1485-1490.

Radlowski EC, Johnson RW (2013) Perinatal iron deficiency and neurocognitive development. Front Hum Neurosci 7:585.

Reinert A, Morawski M, Seeger J, Arendt T, Reinert T (2019) Iron concentrations in neurons and glial cells with estimates on ferritin concentrations. BMC Neurosci 20:25.

Rosato-Siri MV, Badaracco ME, Ortiz EH, Belforte N, Clausi MG, Soto EF, Bernabeu R, Pasquini JM (2010) Oligodendrogenesis in iron-deficient rats: effect of apotransferrin. J Neurosci Res 8:1695-1707.

Rouault TA (2013) Iron metabolism in the CNS: implications for neurodegenerative diseases. Nat Rev Neurosci 14:551-564.

Santiago-González DA, Cheli VT, Zamora NN, Lama TN, Spreuer V, Murphy GG, Paez PM (2017) Conditional deletion of the L-type calcium channel Cav1.2 in NG2 positive cells delay remyelination in mice. J Neurosci 37:10038-10051.

Sanyal B, Szuchet S (1995) Tumor necrosis factor a induces the transcription of the $\mathrm{H}$ chain ferritin gene in cultured oligodendrocytes. Soc Neurosci Abstr 21.

Schonberg DL, Goldstein EZ, Sahinkaya FR, Wei P, Popovich PG, McTigue DM (2012) Ferritin stimulates oligodendrocyte genesis in the adult spinal cord and can be transferred from macrophages to NG2 cells in vivo. J Neurosci 32:5374-5384.

Schulz K, Kroner A, David S (2012) Iron efflux from astrocytes plays a role in remyelination. J Neurosci 32:4841-4847.

Singh AV, Zamboni P (2009) Anomalous venous blood flow and iron deposition in multiple sclerosis. J Cereb Blood Flow Metab 29:1867-1878.

Stephenson E, Nathoo N, Mahjoub Y, Dunn JF, Yong VW (2014) Iron in multiple sclerosis: roles in neurodegeneration and repair. Nat Rev Neurol 10:459-468.
Tarohda T, Yamamoto M, Amamo R (2004) Regional distribution of manganese, iron, copper, and zinc in the rat brain during development. Anal Bioanal Chem 380:240-246.

Todorich B, Pasquini JM, Garcia CI, Paez PM, Connor JR (2009) Oligodendrocytes and myelination: the role of iron. Glia 57:467-478.

Ward RJ, Zucca FA, Duyn JH, Crichton RR, Zecca L (2014) The role of iron in brain ageing and neurodegenerative disorders. Lancet Neurol 13:10451060 .

Williams R, Rohr AM, Wang WT, Choi IY, Lee P, Berman NE, Lynch SG, LeVine SM (2011) Iron deposition is independent of cellular inflammation in a cerebral model of multiple sclerosis. BMC Neurosci 12:59.

Williams R, Buchheit CL, Berman NE, LeVine SM (2012) Pathogenic implications of iron accumulation in multiple sclerosis. J Neurochem 120:725.

Zhang P, Land W, Lee S, Juliani J, Lefman J, Smith SR, Germain D, Kessel M, Leapman R, Rouault TA, Subramaniam S (2005) Electron tomography of degenerating neurons in mice with abnormal regulation of iron metabolism. J Struct Biol 150:144-153.

Zhang Y, Mikhael M, Xu D, Li Y, Soe-Lin S, Ning B, Li W, Nie G, Zhao Y, Ponka P (2010) Lysosomal proteolysis is the primary degradation pathway for cytosolic ferritin and cytosolic ferritin degradation is necessary for iron exit. Antioxid Redox Signal 13:999-1009.

Zhang Y, Chen K, Sloan SA, Bennett ML, Scholze AR, O'Keeffe S, Phatnani HP, Guarnieri P, Caneda C, Ruderisch N, Deng S, Liddelow SA, Zhang C, Daneman R, Maniatis T, Barres BA, Wu JQ (2014) An RNA-sequencing transcriptome and splicing database of glia, neurons, and vascular cells of the cerebral cortex. J Neurosci 34:11929-11947.

Zhu X, Hill RA, Dietrich D, Komitova M, Suzuki R, Nishiyama A (2011) Age-dependent fate and lineage restriction of single NG2 cells. Development 138:745-753. 